\title{
THE UNITARY DUAL FOR THE MULTIPLICATIVE GROUP OF ARBITRARY DIVISION ALGEBRAS OVER LOCAL FIELDS
}

\author{
LAWRENCE CORWIN
}

Let $F$ be a locally compact, nondiscrete, non-Archimedean field (i.e., a finite extension of $\mathbf{Q}_{p}$ or a field of formal Laurent series over a finite field), and let $D$ be a division algebra of degree $n$ over $F$, so that $[D: F]=n^{2}$. In this paper, we give a construction of all irreducible unitary representations of $D^{\times}$. There does not seem to be an easy way to parametrize these representations; Theorem 5.5 is a start in that direction.

The problem of determining $\left(D^{\times}\right)^{-}$is interesting not only in itself, but also for the light it sheds on the problem of determining all irreducible supercuspidal representations of $\mathrm{GL}_{n}(F)$. The problems are connected in two ways. The construction of supercuspidals for $\mathrm{GL}_{n}(F)$ has used procedures quite similar to those used to construct $\left(D^{\times}\right)^{\wedge}$. In previous papers, $\left(D^{\times}\right)^{\wedge}$ has been determined when $p^{2}=n$ or $p^{2} \nmid n$. (See $[11,10,3,4,5]$.) The supercuspidal representations of $\mathrm{GL}_{n}(F)$ are known when $p \nmid n$ and when $n$ is the product of at most two distinct primes (see $[12,17,2,6]$ ), and there is usually a close similarity in methods of construction to the corresponding case for division algebras. Furthermore, one important method of proving that the construction of supercuspidals is complete uses the Matching Theorem of Deligne and Kazhdan (see [8]), which sets up a natural correspondence between $\left(D^{\times}\right)^{\wedge}$ and the set of discrete series representations of $\mathrm{GL}_{n}(F)$. (See, e.g., [2] or [17] for this kind of proof.) It seems likely that the construction in this paper will also apply to give a construction of all supercuspidal representations of $\mathrm{GL}_{n}(F)$. This matter will be dealt with in a future paper. (In this connection, it is worth noting the results in [15] about supercuspidals in $\mathrm{GL}_{n}(F), n$ the product of two primes.)

Describing the general method of the construction requires some notation. Let $k$ be the residue class field of $F$, and suppose that $k$ has $q$ elements. Recall (from, e.g., Chapter I of [18]) that $D$ contains an unramified extension $F_{n}$ of $F$ with $\left[F_{n}: F\right]=n$; the residue class field $k_{n}$ of $F_{n}$, with $q^{n}$ elements, can also be regarded as the residue class field of $D$. Let $R=R_{D}$ be the ring

Received by the editors January 12, 1989 and, in revised form, April 26, 1989.

1980 Mathematics Subject Classification. Primary 22 E50.

The author was supported by NSF Grant DMS-86-03169. 
of integers in $D$ and $P=P_{D}$ its maximal ideal. The solutions in $F_{n}$ to $X^{q^{n}}-X=0$ give representatives for the residue classes of $R / P \cong k_{n}$, and we generally denote this set of coset representatives by $k_{n}$. (This should cause no confusion in the calculations, most of which are modulo $P$.) $D$ also contains a prime element $\varpi$ such that conjugation by $\varpi$ generates $\operatorname{Gal}\left(F_{n} / F\right)$ and such that $\varpi^{n}$ is a prime element in $F$. We write $\varpi x \varpi^{-1}=x^{\sigma}$ for $x \in F_{n}$, and in particular for $x \in k_{n}$. Let $K$ be the group of units in $R$, and write $K^{m}=1+P^{m} \quad(m \geq 1) ; K^{m}$ is normal in $K$ (and in $\left.D^{\times}\right)$, and $K$ is normal in $D^{\times}$. In fact, $D^{\times}=K \times\langle\varpi\rangle$ (semidirect product), and $K / K^{1} \cong k_{n}^{\times}$, $K^{j} / K^{j+1} \cong k_{n}$ via the map $1+\alpha \varpi^{j} \mapsto \alpha$. We sometimes write $K^{0}=K$.

We begin with a character $\chi$ trivial on $K^{m+1}$ but not on $K^{m}$ for some $m$. (The analysis is easy if $\chi$ is trivial on $K$.) We may also assume that $\chi$ does not extend as a character to all of $D^{\times}$. Then $\chi$ can be described by the formula

$$
\chi\left(1+\gamma \varpi^{m}\right)=\psi \circ \operatorname{Tr}_{D / F}\left(\alpha \varpi^{-m} \cdot \gamma \varpi^{m}\right) \quad \forall \gamma \in k_{n}
$$

for some $\alpha \in k_{n}^{\times}$; here $\psi$ is a character of $F$ nontrivial on the ring of integers $R_{F} \subset F$ but trivial on the prime ideal $P_{F}$. (Then $\chi$ also defines a character on $R_{F} / P_{F}=k_{n}$, which we also denote by $\psi$, so that $\chi\left(1+\gamma \varpi^{m}\right)=$ $\psi \circ \operatorname{Tr}_{k_{n} / k}\left(\alpha \gamma^{\sigma^{-m}}\right)$.) From this, we can determine fairly straightforwardly the elements $w \in D^{\times}$such that $\chi\left(w x w^{-1}\right)=\chi(x)$ for all $x \in K^{m}$. (We say that such an element $w$ commutes with $\chi$.) Let $H_{m}$ be the group of commuting $w$.

We next extend $\chi$ to $K^{m-1} \cap H_{m}$ (this group is $K^{m-1}$ unless $m \leq 2$ ), again compute the algebra of commuting elements, and continue inductively. This inductive computation is possible because of two lemmas. One, Lemma 2.2, simplifies the problem of finding the elements commuting with $\chi$. It says that if $w_{0} \in P$ commutes with $\chi$, then so does $1+w_{0} \in K^{1}$. The second, Lemma 3.8 , provides us with a "standard" extension of $\chi$. It says, for instance, that if $\chi$ is defined on $K^{j+1}$, and if both $K^{j}$ and a certain sub-division algebra $D_{0}$ commute with $\chi$, then $\chi$ has an extension $\chi_{0}$ to $K^{j}$ such that $D_{0}$ commutes with $\chi_{0}$. Any other extension of $\chi$ differs from $\chi_{0}$ by a character trivial on $K^{j+1}$ and hence is easily analyzable.

It may be helpful to say something about the connection between the approach used here and that used in the "tame" case (where $p \nmid n$ ). The two methods are surprisingly similar. In each, there are levels $K^{j}$ such that the division algebra $D_{(j)}$ of elements commuting with $\left.\chi\right|_{K^{j}}$ is smaller (in some sense) than that commuting with $\left.\chi\right|_{K^{j+1}}$. (The words "in some sense" are used because the group of elements commuting with $\left.\chi\right|_{K^{j}}$ is not generally a division algebra; however, it contains the nonzero elements of a largest division algebra $D_{(j)}$.) These $j$ are related to the "jump points" described in Koch [13]. In the tamely ramified case, the corresponding $D_{(j)}$ were nested, and one could 
relate $\chi$ to a character of some $E^{\times}$, where $E$ is a field extension of $F$; the $j$ corresponded to the conductors in the Howe factorization of $\chi$ (see [12] or [17]), and there were intermediate fields associated to these $j$ as well. In the general case, the $D_{(j)}$ are not nested, there is no Howe factorization, and the "intermediate fields" are not well defined. What is defined is their ramification index and residue class degree. We say a bit more about this in $\S 6$.

We use the notation of this section throughout the paper, except that we do not use $H_{m}$ for the group of elements commuting with $\left.\chi\right|_{K^{m}}$. We write $\chi^{w}(x)=\chi\left(w x w^{-1}\right)$, but also $\chi^{-1}(x)=(\chi(x))^{-1}$. This should not cause confusion. Lower case Greek letters near the beginning of the alphabet are used for elements of $k_{n}$, and $\eta$ is an element of $P$. The unramified extension of $F$ of degree $d$ and contained in $F_{n}$ is $F_{d}$, and $k_{d}$ is the extension of $k$ with $\left[k_{d}: k\right]=d$.

The bulk of the analysis of $\chi$ is done in $\S 3$; $\S 2$ contains two useful lemmas on commutators. In $\S 4$, we construct the irreducible representations of $D^{\times}$, and in $\S 5$ we show that these exhaust $\left(D^{\times}\right)^{\wedge}$ and compute their degrees. As noted above, $\S 6$ contains a brief comparison of the general case with the tamely ramified case (when $p+n$ ). The final section is devoted to an example.

The recent work that led to this paper was prompted by a question of Allen Moy about the representations of $D_{8}^{\times}\left(\mathbf{Q}_{2}\right)$. I am grateful to him for pushing me to find the answer and for many valuable suggestions about the form and content of this paper. The referee also contributed material improvements in exposition. I am also indebted to Philip Kutzko and David Manderscheid for useful conversations, in which, inter alia, they explained their approach to the problem of supercuspidal representations for $\mathrm{GL}_{n}$.

One final comment. In [14], Helmut Koch referred to a remark made in a paper by Roger Howe and me about "the extremely pleasant geometry of the conjugacy classes in tamely ramified division algebras," and added, "Mir scheint, das man das gleiche auch von dem allgemeinen Fall sagen kann." At the time, I did not see the geometry (or algebra) of $D_{n}^{\times}$as "extremely pleasant" in the general case. Now I realize that his remark was not so unreasonable after all.

We present here two lemmas that will be important in the proof. The first is probably well known; the second may have independent interest.

(2.1) Lemma. Let $x \in K^{m} \cap\left(D^{\times}, D^{\times}\right), m \geq 1$. Then modulo $K^{r+1}(r \geq m)$, $x=\left(u_{m}, v_{m}\right)\left(u_{m+1}, v_{m+1}\right) \cdots\left(u_{r}, v_{r}\right)$, where $v_{j} \in K^{j}$ and each $u_{j}$ is either $\varpi$ or a root of unity in $F_{n}^{\times}$.

Proof. Let $x \equiv 1+\gamma \varpi^{m} \bmod P^{m+1}$. We show that $x \equiv\left(u_{m}, v_{m}\right) \bmod P^{m+1}$ for appropriate $u_{m}, v_{m}$ as above. The lemma then follows by induction. 
If $n \nmid m$, let $\alpha$ be any root of unity generating $F_{n}$ over $F$. Then

$$
\left(\alpha, 1+\beta \varpi^{m}\right) \equiv 1+\left(\alpha \beta\left(\alpha^{\sigma^{m}}\right)^{-1}-\beta\right) \varpi^{m} \bmod P^{m+1} .
$$

But $\alpha \beta\left(\alpha^{\sigma^{m}}\right)^{-1}-\beta=0 \Rightarrow \beta=0$, since $\alpha \neq \alpha^{\sigma^{m}}$. Hence for an appropriate $\beta$, we may make $\alpha \beta\left(\alpha^{\sigma^{m}}\right)^{-1}-\beta=\gamma$, as required.

If $n \mid m$, let $u=\varpi$. Then

$$
\left(\varpi, 1+\beta \varpi^{m}\right) \equiv 1+\left(\beta^{\sigma}-\beta\right) \varpi^{m} \bmod P^{m+1},
$$

so that we can get $(u, v) \equiv x$ if $\operatorname{Tr}_{k_{n} / k} \gamma=0$. However, the character on $K^{m}$ given by

$$
\chi\left(1+\delta \varpi^{m}\right)=\psi \circ \operatorname{Tr}_{k_{n} / k} \delta_{0} \delta, \quad \delta_{0} \in k, \psi \text { as in } \S 1,
$$

extends to $D^{\times}$and is $\neq 1$ (for appropriate $\delta_{0}$ ) if $\operatorname{Tr}_{k_{n} / k} \delta \neq 0$. Thus, if $x$ is a commutator (so that $\chi(x)=1$ ), then $\operatorname{Tr}_{k_{n} / k} \gamma=0$.

Throughout the construction of the representations, we will need to know the group of elements of $D^{\times}$commuting with a character on a normal subgroup. It will simplify matters greatly to know that if an element $\eta \in P$ commutes with a character, so does $1+\eta\left(\in K^{1}\right)$. The following lemma gives what is needed.

(2.2) Lemma. Let $A$ be the ring consisting of all formal power series over $\mathbf{Z}$ in two noncommuting variables $a$ and $b$, with $a^{-1}, b^{-1}$ adjoined. Call an element $x \in A$ integral if the only words in $x$ with nonzero entries are words in $a$ and $b$ (i.e., no negative exponents appear). In the integral elements, let $A_{m}$ be the ideal of words of total exponent $\geq m$ (i.e, the total number of $a$ 's and $b$ 's appearing in each word is $\geq m$ ). Let

$$
\begin{aligned}
x & =(1+a)(1+b)(1+a)^{-1}(1+b)^{-1} \\
& =(1+a)(1+b)\left(1-a+a^{2}-\cdots\right)\left(1-b+b^{2}-\cdots\right),
\end{aligned}
$$

and let $m$ be any positive integer. Then there exist an integer $N$ and elements $c_{j}, d_{j} \in A \quad(1 \leq j \leq N)$ such that

(1) each $c_{j}$ is one of $a, b, a^{-1}, b^{-1}$;

(2) $d_{j}-1 \in A_{1}$;

(3) $c_{j} d_{j} c_{j}^{-1}$ is integral;

(4) the product of commutators $\prod_{j=1}^{N}\left(c_{j}, d_{j}\right)$ differs from $x$ by an element of $A_{m}$.

Proof. Define the weight of a word in the free semigroup on $a$ and $b$ to be $m$ if the total number of $a$ 's and $b$ 's is $m$. Assume inductively that we have elements $c_{j}, d_{j}$ satisfying (1)-(3) with $j \leq N_{0}$ such that if $y_{0}=\prod_{j=1}^{N_{0}}\left(c_{j}, d_{j}\right)$, then $y_{0}^{-1} x$ is a sum $1+\sum_{j} k_{j} w_{j}$, where $k_{j} \in \mathbf{Z}$ and $\sum k_{j} w_{j} \in A_{m}$. It suffices 
to show that we can find more commutators $\left(c_{j}, d_{j}\right), N_{0}<j \leq N_{1}$, satisfying (1)-(3), and such that if $y_{1}=\prod_{j=N_{0}+1}^{N_{1}}\left(c_{j}, d_{j}\right)$, then $y_{1}^{-1} y_{0}^{-1} x \in A_{m+1}$.

Note first that if $w=f_{1} \cdots f_{m}$ (where each $f_{i}$ is an $a$ or a $b$ ), then

$$
\begin{aligned}
\left(f_{1}^{-1}, 1+w\right) & =1+\left(f_{2} \cdots f_{m} f_{1}-f_{1} \cdots f_{m}\right)+\text { terms in } A_{m+1} ; \\
\left(f_{m}, 1+w\right) & =1+\left(f_{m} f_{1} \cdots f_{m-1}-f_{1} \cdots f_{m}\right)+\text { terms in } A_{m+1} .
\end{aligned}
$$

Thus we can use commutators $\left(c_{j}, d_{j}\right), N_{0}+1 \leq j \leq N_{1}$, to replace any word by a cyclic permutation of that word. If this procedure eliminates all words of length $m$, we are done. If not, we produce a contradiction. We may assume that the sum of the terms of weight $m$ is $1=\sum_{j=1}^{r} k_{j} w_{j}$, where each $w_{j}$ is first in alphabetical order among its cyclic permutations and each $k_{j} \in \mathbf{Z}$.

Let $Q$ be a large prime (the rest of the proof will show how large), and let $F=\mathbf{F}_{Q}((X))$, the formal power series field with coefficients in $\mathbf{F}_{Q}$. Let $D$ be the central division algebra of degree $m^{2}$ over $F$, with the Frobenius as generator. Then $D$ is generated by $\mathbf{F}_{Q^{m}}$ and a prime element $\varpi$ with $\varpi^{m}=X$ and $\varpi \gamma \varpi^{-1}=\gamma^{Q}, \forall \gamma \in \mathbf{F}_{Q^{m}}$. We map $A$ onto $D$ by $a \mapsto \alpha \varpi, b \mapsto \varpi$, where $\alpha$ is a primitive $\left(Q^{m}-1\right)$ th root of unity. Then $y_{1}^{-1} y_{0}^{-1} x$ goes to some element $x^{\prime}=1+\gamma_{m} \varpi^{m}+\cdots$, with

$$
\gamma_{m}=\sum_{j=1}^{r} k_{j} \alpha^{l_{j}},
$$

where

$$
l_{j}=\sum\left\{Q^{i-1}: \text { the } i \text { th letter of } w_{j} \text { is an } a\right\} .
$$

Since $x^{\prime}$ is a commutator, it is standard (see the proof of Lemma 2.1) that $\operatorname{Tr}_{\mathbf{F}_{Q^{m} / \mathbf{F}_{Q}}} \gamma_{m}=0$. Thus if we let $\beta_{j}=\alpha^{l_{j}}$ (and choose $Q$ large enough that the $\beta_{j}$ are distinct and nonconjugate as one varies over different words of length $m)$,

$$
\sum_{j=1}^{r} k_{j} \operatorname{Tr}_{\mathbf{F}_{Q^{m} / \mathbf{F}_{Q}}} \beta_{j}=0 .
$$

If we instead map $A$ to $D$ by $a \mapsto \alpha^{s} \varpi, b \mapsto \varpi$, the same argument gives

$$
\sum_{j=1}^{r} k_{j} \operatorname{Tr}_{\mathbf{F}_{Q^{m}} / \mathbf{F}_{Q}} \beta_{j}^{s}=0
$$

Let $\delta_{1}, \ldots, \delta_{t}(t \leq m r)$ be the images of the $\beta_{j}$ under the Galois group. Then

$$
\sum_{j=1}^{m} h_{i} \delta_{i}^{s}=0, \quad 1 \leq s \leq t\left(h_{i}=k_{j} \text { if } \delta_{i} \text { is a conjugate of } \beta_{j}\right) .
$$

Hence the Vandermonde determinant involving the $\delta_{j}$ is 0 , since the columns are linearly dependent (provided that $Q>$ all $h_{i}$ ). On the other hand, this 
determinant is (up to sign)

$$
\prod_{1 \leq i \leq t} \delta_{i} \prod_{1 \leq i<j \leq t}\left(\delta_{i}-\delta_{j}\right) \neq 0,
$$

since the $\beta_{i}$ are nonconjugate. This is the desired contradiction.

(2.3) Remarks. (a) In Lemma 2.2, we may take $u=a$ or $a^{-1}$ whenever the word $v-1$ contains at most one $b$. This is because the $u$ 's are used to permute $v-1$ cyclically, and we can get any cyclic permutation of $a^{m_{1}} b a^{m_{2}}$ by simply shifting $a$ 's.

(b) We typically use Lemma 2.2 in the following way: let $\chi$ be a character on a subgroup $H$ of $K$, and let $s>0$. Assume that $H$ contains all the elements to which we apply $\chi$ in what follows. Suppose that $a \in P, b \in P^{s}$ satisfy $\chi^{a}=\chi$ and $\left.\chi^{b}\right|_{K^{2 s} \cap H}=\left.\chi\right|_{K^{2 s} \cap H}$. Then $\chi((1+a, 1+b))=1$.

Proof. By Lemma 2.2 and the above remark, $\chi(1+a, 1+b)$ is a product of commutators $\left(a, a^{\prime}\right)$ and $\left(b, b^{\prime}\right), a^{\prime} \in H$ and $b^{\prime} \in K^{2 s} \cap H$, and therefore $\chi(a, c)=\chi(b, d)=1$.

We shall abbreviate this argument by saying, "By Lemma 2.2 and Remark $2.3(\mathrm{~b}), \ldots$. .

We now begin the construction of representations of $D^{\times}$. We shall construct all representations with conductor $m+1$; that is, we look at representations trivial on $K^{m+1}$ but not on $K^{m}$. Since we assume inductively that we know all representations with smaller conductor (as well as those for all division algebras of degree $<n$ ), we should begin with the lowest order cases. If $\pi$ is trivial on $K$, then it is defined on $D^{\times} / K \cong \mathbf{Z}$. We then say that $\pi$ has ramification index 1 and residue class degree 1 . If $\pi$ is trivial on $K^{1}$, then it is a representation of $D^{\times} / K^{1} \cong k_{n}^{\times} \times \mathbf{Z}$ (semidirect product), and standard theory determines $\pi$. There is a character $\chi$ on $k_{n}^{\times}$such that $\pi \cong \operatorname{Ind}_{k_{n}^{\times} H}^{D^{\times}} \chi^{\prime}$, where $H$ is the stability group of $\chi$ in $\mathbf{Z}$ and $\chi^{\prime}$ is an extension of $\chi$ to $H$. Each extension gives a different $\pi$, and $\chi_{1}, \chi_{2}$ yield the same set of representations $\pi$ iff $\chi_{1}, \chi_{2}$ are conjugate under $\mathbf{Z}$. If $H=\mathbf{Z}$, then we say that $\pi$ has ramification index 1 and residue class degree 1 . If $[\mathrm{Z}: H]=f>1$, we say that $\pi$ has ramification index 1 and residue class degree $f$, and we associate the triple $(0,1, f)$ to $\pi$.

We now look at the general case. We begin with a character $\chi$ defined on $K^{m}, m \geq 1$, and trivial on $K^{m+1}$. Suppose first that $\chi$ extends to a character $\chi^{\prime}$ of $D^{\times}$. Then any irreducible representation $\pi$ whose restriction in $K^{m}$ contains $\chi$ is of the form $\pi=\pi_{1} \otimes \chi^{\prime}$, where $\pi_{1}$ has smaller conductor than $\pi$. Thus we may regard this case as done. 
We are now at the main case to be considered. We may write

$$
\begin{aligned}
\chi\left(1+\gamma \varpi^{m}\right) & =\psi \circ \operatorname{Tr}_{D / F}\left(\alpha_{1} \varpi^{-m} \gamma \varpi^{m}\right) \\
& =\psi \circ \operatorname{Tr}_{k_{n} / k}\left(\alpha \gamma^{\sigma^{-m}}\right)
\end{aligned}
$$

for some (unique) $\alpha_{1} \in k_{n}$. Furthermore, $\alpha_{1} \varpi^{-m} \notin F$, or else $\chi$ extends to $\chi^{\prime}$ as above. We say that $\chi$ is in general position. Let $m=s_{1}, s_{1}^{\prime}=\left[s_{1} / 2\right]+1$, $s_{1}^{\prime \prime}=s_{1}+1-s_{1}^{\prime}$. Then if $s_{1}=2 m-1$ is odd, we have $s_{1}^{\prime}=s_{1}^{\prime \prime}=m$, while if $s_{1}=2 m$ is even, then $s_{1}^{\prime}=m+1$ and $s_{1}^{\prime \prime}=m$.

We begin by fixing an extension of $\chi$ to $K^{s_{1}^{\prime}}$, which we also call $\chi$. We shall analyze this extension level by level and produce a conjugate with certain desirable properties. We note first that the conjugates under $D^{\times}$of $\left.\chi\right|_{K^{m}}$ are of the form $\chi^{\sim}\left(1+\gamma \varpi^{m}\right)=\psi \circ \operatorname{Tr}_{D / F}\left(\alpha_{1}^{\sim} \varpi^{-m} \gamma \varpi^{m}\right)$, where $\alpha_{1}^{\sim} \varpi^{-m}$ and $\alpha_{1} \varpi^{-m}$ have the same minimal equation. We fix a representative of each conjugacy class and consider only these $\alpha \varpi^{-m}$ in what follows.

(3.1) Theorem. There exist a conjugate of $\chi$ (on $K^{s_{1}^{\prime}}$ ), a sequence $\eta_{\left(s_{1}\right)}$, $\eta_{\left(s_{1}-1\right)}, \ldots, \eta_{\left(s_{1}^{\prime}\right)}$ of elements, a set $s_{1}=m>s_{2}>\cdots>s_{r_{1}}$ of distinguished indices, and a set of pairs of integers $\left(e_{i}, f_{i}\right), 1 \leq i \leq r_{1}$, with the following properties (in what follows, for an index $j, i$ is the largest index with $s_{i} \geq j$, and similarly for $j^{\prime}, i^{\prime}$; we suppose that $\chi$ itself is the desired conjugate of $\chi$ ):

(1) The group of elements of $D^{\times}$commuting with $\left.\chi\right|_{K^{j}}$ is

$$
K^{s_{1}-j+1}\left(K^{s_{2}-j+1} \cap D_{1}^{\times}\right) \cdots\left(K^{s_{i}-j+1} \cap D_{i-1}^{\times}\right) D_{(j)}^{\times}
$$

where, for arbitrary indices $j^{\prime}$ and $h, D_{\left(j^{\prime}\right)}$ is the division algebra generated over $F$ by $\eta_{\left(j^{\prime}\right)}$ and $k_{n / e_{i^{\prime}}}$, and $D_{h}=D_{\left(s_{h+1}+1\right)}, \eta_{h}=\eta_{\left(s_{h+1}+1\right)}$, etc.

(2) The center of $D_{(j)}$ is an extension $E_{(j)}$ of $F$ with ramification index $e_{i}$ and maximal unramified extension $F_{f_{i}} ; D_{(j)}$ has index $n / e_{i} f_{i}$ over $E_{(j)}$ (i.e., its dimension over $E_{(j)}$ is $\left.\left(n / e_{i} f_{i}\right)^{2}\right)$ and it contains $F_{n / e_{i}}$.

(3) $\eta_{(j)} \gamma \eta_{(j)}^{-1}=\gamma^{\sigma^{f_{i}}}$ for $\gamma \in k_{n / e_{i}}$.

(4) $\eta_{(j)}$ is a generator of $P^{f_{i}}$, and $\eta_{(j)}^{n / e_{i} f_{i}}$ generates $E_{(j)}$ over $F_{f_{i}}$.

(5) $e_{i-1}\left|e_{i}, f_{i-1}\right| f_{i}$, and $e_{i} f_{i} \mid n ; e_{i-1} f_{i-1}<e_{i} f_{i}$ and $e_{1} f_{1}>1$.

(6) $e_{i}=n /\left(n / e_{i-1}, s_{i}\right)$, where $e_{0}=1$ and $($,$) is the greatest common$ divisor.

(7) (i) Suppose that $j_{1}<j$ and $f_{i} \mid j_{1}$. Let $\psi_{0}$ be any character of $k_{n / e_{i-1}}$ trivial on $k_{n / e_{i}}$. Then there exists $\delta \in k_{n / e_{i-1}}$ such that if we set

$$
w=1+\eta_{i-1}^{\left(s_{i}-j_{1}\right) / f_{i-1}}, \quad \chi^{\#}(y)=\chi\left(w y w^{-1} y^{-1}\right),
$$

then $\chi^{\#}$ is trivial on $P^{j_{1}+1}$ and $\chi^{\#}\left(1+\gamma \eta_{(j)}^{j_{1}}\right)=\psi_{0}(\gamma), \forall \gamma \in k_{n / e_{i-1}}$. convention, $\eta_{0}=\varpi$.) 
(ii) If $j_{1}<j$ and $f_{i}+j_{1}$ but $f_{i-1} \mid j_{1}$, then for any character $\psi_{0}$ of $k_{n / e_{i-1}}$ there exists $\delta \in k_{n / e_{i-1}}$ such that if $w, \chi^{\#}$ are defined as in (3.2), then $\chi^{\#}$ is trivial on $P^{j_{1}+1}$ and $\chi^{\#}\left(1+\gamma \eta_{(j)}^{j_{1}}\right)=\psi_{0}(\gamma), \forall \gamma \in k_{n / e_{h}}$.

(8) $D_{(j)}^{\times} \cap K^{r} \subseteq\left(D_{(j+1)}^{\times} \cap K^{r}\right)\left(D_{i-1}^{\times} \cap K^{r+s_{i}-j}\right) \cdots\left(D_{1}^{\times} \cap K^{r+s_{2}-j}\right) K^{r+s_{1}-j}$ and

$$
D_{(j)} \cap P^{r} \subseteq\left(D_{(j+1)} \cap P^{r}\right)+\left(D_{i-1} \cap P^{r+s_{i}-j}\right)+\cdots+P^{r+s_{1}-j} .
$$

(Note. For this theorem, we take $s_{r_{1}+1}=s^{\prime}-1$.)

(9) $\eta_{(j)} \equiv \eta_{i} \bmod P^{f_{i}+1}$ if $i$ is the largest index with $j \leq s_{i}$; if $j=s_{i}, \eta_{(j)}$ is congruent $\bmod P^{f_{i}+1}$ to an element of $D_{i}$.

We will add a tenth property; see (3.9) infra.

(3.3) Notes. (1) We will associate a sequence of triples with $\chi$; the sequence will begin with $\left(s_{1}, e_{1}, f_{1}\right), \ldots,\left(s_{r_{1}}, e_{r_{1}}, f_{r_{1}}\right)$. Further details will be given in $\S 4$.

(2) It may help the reader to have the following explanation of the properties in Theorem 3.1. In producing an irreducible from $\chi$, Clifford-Mackey theory shows that we need to know $\left\{x: \chi^{x}=\chi\right\}$; this information is given by property (1). Properties (2)-(4) describe the structure of $D_{(j)}$. They show that it is generated over its center $E_{(j)}$ by $k_{n / e_{i}}$ and $\eta_{(j)}$ exactly as $D_{n}$ is generated over $F$ by $k_{n}$ and $\varpi$. Properties (5) and (6) characterize the sequences $\left\{\left(s_{i}, e_{i}, f_{i}\right)\right\}$ associated with irreducible representations of $D^{\times}$. Property (7) describes the freedom we have in conjugating $\chi$ into a more tractable form. Property (8) shows that the terms in the product for (1) fit well with one another. It implies, for instance, that $K^{s_{1}-j+1} \cdots\left(K^{s_{h}-j+1} \cap D_{h-1}^{\times}\right)$is normal in the group of elements commuting with $\left.\chi\right|_{K^{j}}$. Finally, (9) says, e.g., that if $j \neq s_{i}$, then $D_{(j)} \cap P^{r}$ and $D_{(j+1)} \cap P^{r}$ give the same residue classes $\bmod P^{r+1}$.

(3) On $K^{s_{1}^{\prime}}, \chi$ is given by $\chi(1+y)=\Psi \circ \operatorname{Tr}_{D / K}(x y)$ for some $x \in$ $P^{-s_{1}}\left(\bmod P^{1-s_{1}^{\prime}}\right)$, and the theorem could be proved by analyzing the elements that commute with $x$ (or a suitable conjugate of $x$ ) modulo an appropriate power of $P$. Such a proof, however, would not apply to the extensions of Theorem 3.1 that we will need in $\S 4$. We give a proof that will apply inductively to these extensions.

The proof is long; we divide it into parts for future reference.

(3.4) We begin be verifying everything for $j=m=s_{1}$. By hypothesis, $\alpha_{1} \varpi^{-m} \notin F$. Let $F\left[\alpha_{1} \varpi^{-m}\right]=E_{\left(s_{1}\right)}$ have ramification index $e_{1}$ and residue class degree $f_{1}$ over $F$. Then $e_{1} f_{1}>1$, and $e_{1}(n, m)=n$ (since $e_{1}=$ $n /(n, m)$ is the smallest positive exponent such that $\left(\alpha_{1} \varpi^{-m}\right)^{e_{1}}$ generates an ideal $P^{r}$ with $n$ dividing $r$ ). Let $D_{\left(s_{1}\right)}$ be the division algebra of elements 
commuting with $E_{\left(s_{1}\right)}$. We now prove property (1). Since the commutator subgroup $\left(K^{1}, K^{m}\right) \subseteq K^{m+1} \subseteq \operatorname{Ker} \chi, K^{1}$ commutes with $\chi$. If $w \in D_{\left(s_{1}\right)}^{\times}$, then for $y=1+y_{0}$, we have

$$
\begin{aligned}
\chi^{w}(y) & =\chi\left(w y w^{-1}\right)=\psi \circ \operatorname{Tr}_{D / F}\left(\alpha_{1} \varpi^{-m} \cdot w y_{0} w^{-1}\right) \\
& =\psi \circ \operatorname{Tr}_{D / F}\left(w^{-1} \cdot \alpha_{1} \varpi^{-m} \cdot w y_{0}\right) \\
& =\psi \circ \operatorname{Tr}_{D / F}\left(\alpha_{1} \varpi^{-m} y_{0}\right)=\chi(y),
\end{aligned}
$$

since $\left[\alpha_{1} \varpi^{-m}, w\right]=0$. Thus all elements in $K^{1} D_{\left(s_{1}\right)}^{\times}$commute with $\chi$.

Conversely, suppose that $\chi^{w}=\chi$, with $w=\delta \varpi^{h_{0}}\left(1+\delta_{1} \varpi+\cdots\right)$. Since $1+\delta_{1} \varpi+\delta_{2} \varpi^{2}+\cdots=w_{1}$ satisfies $\chi^{w_{1}}=\chi$, it suffices to consider $w=\delta \varpi^{h}$. Writing $y$ as above, we have

$$
\psi \circ \operatorname{Tr}_{D / F}\left(\alpha_{1} \varpi^{-m} \gamma \varpi^{m}\right)=\psi \circ \operatorname{Tr}_{D / F}\left(\alpha_{1} \varpi^{-m} \cdot \delta \varpi^{h} \cdot \gamma \varpi^{m} \cdot\left(\delta \varpi^{h}\right)^{-1}\right),
$$

or

$$
\psi \circ \operatorname{Tr}_{k_{n} / k}\left(\alpha_{1} \gamma^{\sigma^{-m}}\right)=\psi \circ \operatorname{Tr}_{k_{n} / k}\left(\alpha_{1} \delta^{\sigma^{-m}} \gamma^{\sigma^{h-m}} \delta^{-1}\right),
$$

for all $\gamma \in k_{n}$. Equivalently,

$$
\psi \circ \operatorname{Tr}_{k_{n} / k} \gamma\left(\alpha_{1}^{\sigma^{m}}-\alpha_{1}^{\sigma^{m-h}} \delta^{\sigma^{-h}}\left(\delta^{\sigma^{m-h}}\right)^{-1}\right)=1, \quad \forall \gamma \in k_{n} .
$$

Hence $\delta^{\sigma^{m-h}} \alpha_{1}^{\sigma^{m}}=\alpha_{1}^{\sigma^{m-h}} \delta^{\sigma^{-h}}$, or $\delta \alpha^{\sigma^{h}}=\alpha \delta^{\sigma^{-m}}$, and this implies that

$$
\left[\delta \varpi^{h}, \alpha_{1} \varpi^{-m}\right]=0 \text {. }
$$

Therefore $\delta \varpi^{h} \in D_{\left(s_{1}\right)}^{\times}$.

(3.5) Since $\left(\alpha_{1} \varpi^{-m}\right)^{e_{1}}=\alpha^{\prime} \varpi^{-r n}$ for some $r$, we see that $F_{f_{1}}$ is the maximal unramified extension of $E_{\left(s_{1}\right)}$. Let $\beta \varpi^{n / e_{1}}$ be a prime element in $E_{\left(s_{1}\right)}$, with $\beta \in k_{n}$. Then $\beta \varpi^{n / e_{1}}$ has an $\left(n / e_{1} f_{1}\right)$ th root $\eta_{\left(s_{1}\right)}$ in $D$, since

$$
\left[E_{s_{1}}\left(\left(\beta \varpi^{n / e_{1}}\right)^{e_{1} f_{1} / n}\right): F\right]=n .
$$

It is clear that $\eta_{\left(s_{1}\right)}$ generates $P^{f_{1}}$. Since $\eta_{\left(s_{1}\right)}$ is of the form $\beta^{\prime} \varpi^{f_{1}}$, (3) clearly holds. We have also verified (4)-(6), and (8) and (9) are vacuous. $D_{\left(s_{1}\right)}$ contains $\eta_{\left(s_{1}\right)}$ and $k_{n / e_{1}}$, and these elements generate an algebra of dimension $n^{2} / e_{1}^{2} f_{1}^{2}$ over $E_{\left(s_{1}\right)}$. Therefore they generate $E_{\left(s_{1}\right)}$, and (3) holds. Thus we need check only (7).

(3.6) Let $j_{0}$ satisfy $s_{1}^{\prime} \leq j_{0}<s_{1}$; set $w=1+\delta \varpi^{s_{1}-j_{0}}$. Then for $y=1+\gamma \varpi^{j_{0}}$, we have

$$
\begin{aligned}
\chi\left(w y w^{-1} y^{-1}\right) & =\chi\left(1+\left(\delta \gamma^{\sigma^{s_{1}-j_{0}}}-\gamma \delta^{\sigma_{0}}\right) \varpi^{s_{1}}\right) \\
& =\psi \circ \operatorname{Tr}_{k_{n} / k} \alpha_{1}\left(\delta \gamma^{\sigma^{s_{1}-j_{0}}}-\gamma \delta^{\sigma_{0} j_{0}}\right)^{\sigma^{-s_{1}}} \\
& =\psi \circ \operatorname{Tr}_{k_{n} / k} \gamma\left(\delta^{\sigma_{j_{0}-s_{1}}} \alpha_{1}^{\sigma^{j_{0}}}-\delta^{\sigma^{j_{0}}} \alpha_{1}^{\sigma^{s_{1}}}\right)
\end{aligned}
$$


while $w y w^{-1} y^{-1} \in K^{s_{1}+1}$ for $y \in K^{j_{0}+1}$. Hence $\chi\left(w y w^{-1} y^{-1}\right)$ is trivial on $K^{j_{0}+1}$.

Suppose first that $f_{1}+j_{0}$. Then the above character is never trivial for $\delta \neq 0$; if it were, then we would have to have

$$
\begin{gathered}
0=\delta^{\sigma^{j_{0}-s_{1}}} \alpha_{1}^{\sigma^{j_{0}}}-\delta^{\sigma^{j_{0}}} \alpha_{1}^{\sigma^{s_{1}}}, \quad \delta^{\sigma^{-s_{1}}} \alpha_{1}=\alpha_{1}^{\sigma^{s_{1}-j_{0}}} \delta \\
{\left[\alpha \varpi^{-s_{1}}, \delta \varpi^{s_{1}-j_{0}}\right]=0,}
\end{gathered}
$$

and this is impossible because $D_{\left(s_{1}\right)}$ does not contain a generator of $P^{s_{1}-j_{0}}$. Hence we obtain every character of $k_{n}$ by varying $\delta$, and this proves (7)(ii). If $f_{1} \mid j_{0}$, the same calculation shows that the above character is trivial whenever $w=1+\delta^{\prime} \eta_{\left(s_{1}\right)}^{\left(s_{1}-j_{0}\right) / f_{1}}, \delta^{\prime} \in k_{n / e_{1}}$. (These are the elements commuting with $\alpha_{1} \varpi^{-s_{1}}$.) Thus we obtain $\operatorname{Card}\left(k_{n} / k_{n / e_{1}}\right)$ distinct $\chi$. On the other hand, if $y=1+\gamma_{0} \eta_{\left(s_{1}\right)}^{j_{0} / f_{1}}$, with $\gamma_{0} \in k_{n / e_{1}}$, then (writing $w=1+\delta_{0} \eta_{\left(s_{1}\right)}^{\left(s_{1}-j_{0}\right) / f_{1}}, \delta_{0} \in k_{n}$, and $\alpha_{1} \varpi^{-m}=\alpha_{0} \eta_{\left(s_{1}\right)}^{s_{1} / f_{1}} ; \alpha_{0} \in k_{f_{1}}$ because $\alpha_{1} \varpi^{-m}$ and $\eta_{\left(s_{1}\right)}^{-s_{1} / f_{1}}$ are in $\left.E_{\left(s_{1}\right)}\right)$ :

$$
\begin{aligned}
\chi\left(w y w^{-1} y\right)^{-1} & =\chi\left(1+\left(\delta_{0} \gamma_{0}^{\sigma^{s_{1}-j_{0}}}-\gamma_{0} \delta_{0}^{\sigma^{j_{0}}}\right) \eta_{\left(s_{1}\right)}^{s_{1} / f}\right) \\
& =\psi \circ \operatorname{Tr}_{k_{n} / k} \delta_{0} \alpha_{0}\left(\gamma_{0}^{\sigma_{1}^{s_{1}-j_{0}}}-\gamma_{0}^{\sigma^{-j_{0}}}\right),
\end{aligned}
$$

since $f_{1} \mid j_{0}$ and $f_{1} \mid s_{1}$. Furthermore, $\left(n / e_{1}\right) \mid s_{1}$, so that

$$
\operatorname{Tr}_{k_{n} / k_{n / e_{1}}} \delta_{0} \alpha_{0}\left(\gamma_{0}^{\sigma^{s_{1}-j_{0}}}-\gamma_{0}^{\sigma^{-j_{0}}}\right)=\delta_{0} \alpha_{0} \operatorname{Tr}_{k_{n} / k_{n / e_{1}}}\left(\gamma_{0}^{\sigma^{s_{1}}}-\gamma\right)^{\sigma^{-j_{0}}}=0
$$

Hence $\chi\left(w y w^{-1} y^{-1}\right)=1$ for all $y$ as above. Since there are $\operatorname{Card}\left(k_{n} / k_{n / e_{1}}\right)$ characters of $k_{n}$ fixing $k_{n / e_{1}}$, we see that we can obtain exactly these characters by varying $\delta$. This proves (7)(i).

In the inductive step, we begin with $\left.\chi\right|_{K^{j+1}}$ and consider $\left.\chi\right|_{K^{j}}$. It will be useful to compare our character $\left.\chi\right|_{K^{j}}$ with a "best possible" extension of $\left.\chi\right|_{K^{j+1}}$ to $K^{j}$. The next lemma describes this extension.

(3.8) Lemma. Let $\chi$ be a character on $K^{j+1}, s_{1}^{\prime} \leq j<s_{1}$, and let $e, f$ be integers with $e f \mid n$ such that both $k_{n / e}^{\times}$and an element $\eta$ generating $P^{f}$ commute with $\chi$. Assume also that $\eta \alpha \eta^{-1}=\alpha^{\sigma^{f}}$ for all $\alpha \in k_{n / e}$. Then $\chi$ has an extension $\chi_{0}$ to $K^{j}$ such that $k_{n / e}$ and $\eta$ commute with $\chi_{0}$.

Proof. We consider two cases separately.

(a) Assume that $(n / e) \nmid j$. There are $|k|^{n}$ extensions of $\chi$ to $K^{j}$, since $\left[K^{j}: K^{j+1}\right]=|k|^{n}$ and $K^{j} / K^{s_{1}+1}$ is Abelian. These extensions form an affine space $V$, so that if $\chi_{1}, \chi_{2}, \chi_{3}$ are any three extensions, then $\chi_{1} \chi_{2} \chi_{3}^{-1}$ is also an extension. Furthermore, at most one extension $\chi_{0}$ is fixed by $k_{n / e}^{\times}$. For if $\chi_{0}, \chi_{0}^{\prime}$ are both $k_{n / e}^{\times}$-fixed, then $\chi_{0}\left(\chi_{0}^{\prime}\right)^{-1}=\chi^{\sim}$ is a nontrivial character 
on $K^{j}$, trivial on $K^{j+1}$ and fixed by $k_{n / e}^{\times}$. We have

$$
\chi^{\sim}\left(1+\gamma \varpi^{j}\right)=\psi \circ \operatorname{Tr}_{k_{n} / k}\left(\delta \gamma^{\sigma^{-j}}\right), \quad \forall \gamma \in k_{n},
$$

for some $\delta \in k_{n / e}$. Since $\beta\left(1+\gamma \varpi^{j}\right) \beta^{-1}=1+\gamma \beta\left(\beta^{-1}\right)^{\sigma^{j}} \varpi^{j}$, we get

$$
\psi \circ \operatorname{Tr}_{k_{n} / k}\left(\delta \gamma^{\sigma^{-j}}\right)=\psi \circ \operatorname{Tr}_{k_{n} / k}\left(\delta \beta^{\sigma^{-j}} \beta^{-1} \gamma^{\sigma^{-j}}\right), \quad \forall \gamma \in k_{n} \quad \text { and } \quad \forall \beta \in k_{n / e} .
$$

So $\delta \beta=\delta \beta^{\sigma^{j}}, \forall \beta \in k_{n / e}$, and $\delta=0$ because $j$ is not a multiple of $n / e$.

Consider the $k_{n / e^{\times}}^{\times}$-orbits in $V$. Since $|k|^{n}$ is a power of $p$, while $\left|k_{n / e}^{\times}\right|$is one less than a power of $p$, it is clear that the greatest common divisor of the cardinalities of orbits is 1 . Hence we can find orbits $\mathscr{O}_{1}, \ldots, \mathscr{O}_{r}$ and integers $h_{1}, \ldots, h_{r}$ with $\sum_{i=1}^{r} h_{i}\left|\mathscr{O}_{i}\right|=1$. Let

$$
\chi_{0}=\prod_{i=1}^{r} \prod_{\chi^{\prime} \in \mathscr{O}_{i}}\left(\chi^{\prime}\right)^{h_{i}} .
$$

Then $\chi_{0}$ extends $\chi$ and is obviously $k_{n / e}^{\times}$-stable. Since $\eta$ normalizes $k_{n / e}$, $\chi_{0}^{\eta}(x)=\chi_{0}\left(\eta \times \eta^{-1}\right)$ is also $k_{n / e}^{\times}$-stable. From what we proved above, $\chi_{0}^{\eta}=\chi_{0}$. Therefore $\chi_{0}$ is the required extension.

(b) Suppose that $(n / e) \mid j$. Then $\delta\left(1+\gamma \eta^{j / f}\right) \delta^{-1}=1+\gamma \eta^{j / f}$ for all $\gamma \in k_{n}$ and all $\delta \in k_{n / e}^{\times}$. Thus every extension of $\chi$ to $K^{j}$ is fixed by $k_{n / e}^{\times}$. Let $\chi_{1}$ be any extension of $\chi$. Then every extension is of the form $\chi_{\zeta}=\chi_{1} \psi_{\zeta}$, where

$$
\psi_{\zeta}\left(1+\gamma \eta^{j / f}\right)=\psi \circ \operatorname{Tr}_{k_{n} / k}(\zeta \gamma), \quad \forall \gamma \in k_{n},
$$

for some $\zeta \in k_{n}$. Write $\eta=\beta \varpi^{f}+\cdots$ and let $y=1+\gamma \eta^{j / f}$. A calculation gives

$$
\begin{aligned}
\psi_{\zeta}\left(\eta y \eta^{-1}\right) \psi_{\zeta}(y)^{-1} & =\psi \circ \operatorname{Tr}_{k_{n} / k} \zeta\left(\gamma^{\sigma^{f}}-\gamma\right) \\
& =\psi \circ \operatorname{Tr}_{k_{n} / k} \gamma\left(\zeta-\zeta^{\sigma^{-f}}\right) .
\end{aligned}
$$

This shows first that $\psi_{\zeta}\left(\eta y \eta^{-1}\right)=\psi_{\zeta}(y)$ if $\gamma \in k_{f}$ and then that $\psi_{\zeta}^{\eta}=\psi_{\zeta}$ iff $\zeta \in k_{f}$. Hence as $\zeta$ runs through $k_{n}$, the characters $\psi_{\zeta}^{\eta}\left(\psi_{\zeta}\right)^{-1}$ run through all characters of $K^{j}$ trivial on $K^{j+1}$ and on the elements $1+\gamma \eta^{j / f}, \gamma \in k_{f}$.

Now consider $\chi_{1}^{\eta}(x) \chi_{1}(x)^{-1}$. For $x \in K^{j+1}$, this is 1 by hypothesis. If $x=1+\gamma \eta^{j / f}$ with $\gamma \in k_{f}$, then $[x, \eta]=0$, and $\chi_{1}^{\eta}(x) \chi_{1}(x)^{-1}=1$. So $\chi_{1}^{\eta}\left(\chi_{1}\right)^{-1}=\left(\psi_{\zeta}^{\eta}\left(\psi_{\zeta}\right)^{-1}\right)^{-1}$ for some $\zeta$, and $\chi_{1} \psi_{\zeta}$ is then $\eta$-stable.

We make the notational convention at each step of the inductive process that $\chi$ is the given representation on $K^{j}$ and $\chi_{0}$ is an extension of $\left.\chi\right|_{K^{j+1}}$ to $K^{j}$ with the property of the lemma (with respect to $\eta_{(j+1)}$ and $k_{n / e_{i}}$ ). 
(3.9) We now make our final inductive assumption, as promised earlier:

(10) For $j=s_{i}$ (so that $\eta_{(j+1)}=\eta_{i-1}$, etc.),

$$
\chi \chi_{0}^{-1}\left(1+\gamma \eta_{i-1}^{s_{i} / f_{i-1}}\right)=\psi \circ \operatorname{Tr}_{k_{n} / k} \alpha_{i}^{\prime} \gamma^{\sigma^{s_{i}}}
$$

where, if $\alpha_{i}=\operatorname{Tr}_{k_{n} / k_{n / e_{i-1}}} \alpha_{i}^{\prime}$, then $E_{i-1}\left[\alpha_{i} \eta_{i-1}^{s_{i} / f_{i-1}}\right]$ is a field of ramification index $e_{i}$ and residue class degree $f_{i}$ over $F ; \eta_{(j-1)} \equiv \delta \eta_{i-1}^{f_{i} / f_{i-1}} \bmod P^{f_{i}+1}$, where $\delta$ commutes with $E_{i-1}\left[\alpha_{i} \eta_{i-1}^{s_{i} / f_{i-1}}\right]$.

For $i=1$, we have $e_{0}=f_{0}=1$ and we take $\eta_{0}=\varpi, F_{0}=F$. It is easy to check that (10) holds in this case.

(3.10) It may be useful to see one early step in the induction worked out before we go to the general step. Assume that for $j+1 \leq h \leq s_{1}$, we have $\eta_{(h)}=\eta_{\left(s_{1}\right)}$ and $D_{(h)}=D_{\left(s_{1}\right)}$. We assume that (1)-(10) hold for $h \geq j+1$ (so that we are not yet at $s_{2}$ ), and examine what occurs at level $j$. Let $\chi_{0}$ be the extension of $\left.\chi\right|_{K^{j+1}}$ with the properties of Lemma 3.8. If $f_{1} \nmid j$, then $\chi$ is conjugate to $\chi_{0}$ by an element fixing $\left.\chi\right|_{K^{j+1}}$, from (7)(ii). Thus we may assume that $\chi=\chi_{0}$. Let $\eta_{(j)}=\eta_{\left(s_{1}\right)}$ and $D_{(j)}=D_{\left(s_{1}\right)}$. Then $s_{2}<j$. Properties (2)-(10) are now all obvious for $j$; (1) requires a calculation, which is omitted because a similar calculation will occur at the end of case (a) below.

So assume that $f_{1} \mid j, j=j_{0} f_{1}$. Then $\chi^{\sim}=\chi \cdot \chi_{0}^{-1}$ is a character trivial on $K^{j+1}$, and on $K^{j}$ there is a unique $\alpha_{2}^{\prime} \in k_{n}$ such that

$$
\begin{aligned}
\chi^{\sim}\left(1+\gamma \eta_{(j+1)}^{j_{0}}\right) & =\psi \circ \operatorname{Tr}_{D / F}\left(\alpha_{2}^{\prime} \eta_{(j+1)}^{-j_{0}} \cdot \gamma \eta_{(j+1)}^{j_{0}}\right) \\
& =\psi \circ \operatorname{Tr}_{k_{n} / k}\left(\alpha_{2}^{\prime} \gamma^{\sigma^{-j}}\right)
\end{aligned}
$$

for all $\gamma \in k_{n}$. Let $\alpha_{2}=\operatorname{Tr}_{k_{n} / k_{n / e_{1}}}\left(\alpha_{2}^{\prime}\right)$. There are now two cases to consider.

(a) Assume that $\alpha_{2} \eta_{(j+1)}^{-j_{0}} \in E_{(j+1)}\left(=E_{\left(s_{1}\right)}\right.$, by assumption). Note that since $\eta^{n / e_{1} f_{1}}$ is prime in $E_{(j+1)}$, we have $\left(n / e_{1}\right) \mid j_{0}$. Let $\beta$ be a fixed element of $k_{n}$ such that $\operatorname{Tr}_{k_{n} / k_{n / e_{1}}} \beta=1$ (if $\left(e_{1}, p\right)=1, \beta$ can be in the prime field). Then $\operatorname{Tr}_{k_{n} / k_{n / e_{1}}} \alpha_{2} \beta=\alpha_{2}=\operatorname{Tr}_{k_{n} / k_{n / e_{1}}} \alpha_{2}^{\prime}$. In view of (7)(i), we may assume that by conjugation that $\alpha_{2} \beta=\alpha_{2}^{\prime}$. In particular, we may assume $\alpha_{2}^{\prime}=0$ if $\alpha_{2}=0$. Also, $\alpha_{2} \in k_{n} \cap E_{(j+1)}=k_{f_{1}}$ because $\alpha_{2} \eta_{(j+1)}^{-j_{0}}$ and $\eta_{(j+1)}^{-j_{0}}$ are in $E_{(j+1)}$.

We first define $\eta_{(j)}$ to be $\eta_{(j+1)}\left(1+\delta \varpi^{t}\right)$, where $t=s_{1}-j$ and $\delta$ is to be determined. We want $\chi^{\eta_{(j)}}=\chi$ on $K^{j}$. On $K^{j+1}$ this holds by property (1). On $K^{j}$, we have $\chi_{0}^{\eta_{(j)}}=\chi_{0}^{\left(1+\delta \varpi^{t}\right)}$, since $\chi_{0}^{\eta_{(j+1)}}=\chi_{0}$, while $\left(\chi^{\sim}\right)^{\eta_{(j)}}=$ $\left(\chi^{\sim}\right)^{\eta_{(j+1)}}$ because conjugation by $1+\delta \varpi^{t}$ is trivial $\bmod K^{j+1}$. (This follows from Lemma 2.2 and Remark 2.3(b).) Hence

$$
\chi^{\eta_{(j)}}=\chi_{0}^{\left(1+\delta \varpi^{t}\right)}\left(\chi^{\sim}\right)^{\eta_{(j+1)}} .
$$


But $\left(\chi^{\sim}\right)^{\eta_{(j+1)}}\left(\chi^{\sim}\right)^{-1}$ is trivial on the elements $1+\gamma \eta_{(j+1)}^{j_{0}}, \gamma \in k_{n / e_{1}}$, since

$$
\begin{aligned}
\left(\chi^{\sim}\right)^{\eta_{(j+1)}}\left(\chi^{\sim}\right)^{-1}\left(1+\gamma \eta_{(j+1)}^{j_{0}}\right) & =\chi^{\sim}\left(1+\left(\gamma^{\sigma^{j}}-\gamma\right) \eta_{(j+1)}^{j_{0}}\right) \\
& =\psi \circ \operatorname{Tr}_{k_{n / e_{1}} / k} \alpha_{2}\left(\gamma^{\sigma^{j}}-\gamma\right)^{\sigma^{-j}} \\
& =\psi \circ \operatorname{Tr}_{k_{n / e_{1}} / k}\left(\left(\alpha_{2} \gamma\right)^{\sigma^{j}}-\alpha_{2} \gamma\right)^{\sigma^{-j}}=1
\end{aligned}
$$

(recall that $\left.\alpha_{2} \in k_{f}\right)$. From (7)(i), we can choose $\delta$ so that $\left(\chi_{0}^{\left(1+\delta \varpi^{t}\right)}\right)^{-1} \chi_{0}=$ $\left(\chi^{\sim}\right)^{\eta_{(j+1)}}\left(\chi^{\sim}\right)^{-1}$, and this is the desired $\delta$.

Because $t$ is a multiple of $n / e_{1}, 1+\delta \varpi^{r}$ commutes with $k_{n / e_{1}}$. Thus (3) follows by the inductive hypothesis. Clearly $\eta_{(j)}^{n / e_{1} f_{1}}$ is central in $D_{(j)}$. Since $\left[F_{f_{1}}\left[\eta_{(j)}^{n / e_{1} f_{1}}\right]: F\right] \geq e_{1} f_{1}$ and $\left[D_{(j)}: F\right]=n^{2} / e_{1} f_{1}$, we must have $\left[F\left[\eta_{(j)}^{n / e_{1} f_{1}}\right]: F\right]$ $=e_{1} f_{1}$. Hence $F\left[\eta_{(j)}^{n / e_{1} f_{1}}\right]=E(j)$. The rest of (2) is now easy, and we have also checked (4); (5), (6), (7), and (10) are true because the claim is exactly the same as for $j+1$. It is also easy to check (8) and (9).

That leaves property (1). $D_{(j)}^{\times}$commutes with $\chi$ by Lemma 2.2 , and $K^{s_{1}-j+1}$ commutes with $\chi$ because $\left(K^{s_{1}-j+1}, K^{j}\right) \subseteq K^{s_{1}+1}$. Conversely, any element $w$ commuting with $\chi$ commutes with $\left.\chi\right|_{K^{j+1}}$. Therefore

$w=\delta_{0} \eta_{(j+1)}^{h}\left(1+\delta_{1} \eta_{(j+1)}+\cdots+\delta_{r-1} \eta_{(j+1)}^{r-1}+\delta_{s_{1}-j} \varpi^{s_{1}-j}+\delta_{s_{1}-j+1} \varpi^{s_{1}-j+1}+\cdots\right)$, where $f_{1} r=s_{1}-j, \delta_{l} \in k_{f_{1}}$ if $l \leq r+1$, and $\delta_{l} \in k_{n}$ for $l \geq r$. (Note the jump in indices between $r-1$ and $s_{1}-j$.) Because of our expression for $\eta_{(j)}$, we can replace each $\eta_{(j+1)}$ by $\eta_{(j)}$ at the cost of changing the $\delta_{l}$ with $l \geq r$. Similarly, we can replace $\varpi^{s_{1}-j}$ with $\eta_{(j)}^{r}$ by again changing the $\delta_{l}$ with $l \geq r$. Thus we may write

$$
w=\delta_{0} \eta_{(j)}^{h}\left(1+\delta_{1} \eta_{(j)}+\cdots+\delta_{r} \eta_{(j)}^{r}+\delta_{s_{1}-j+1} \varpi^{s_{1}-j+1}+\delta_{s_{1}-j+2} \varpi^{s_{1}-j+1} \cdots\right),
$$

where the $\delta_{i}$ are in $k_{f_{1}}$ for $i<r$ and are in $k_{n}$ thereafter. Dividing by an element known to commute with $\chi$, we consider $w=1+\delta_{r} \eta_{(j)}^{r}$. We need $\delta_{r} \in k_{n / e_{1}}$.

Suppose that $\delta_{r} \eta_{(j)}^{r}=\delta \varpi^{s_{1}-j}+\cdots$. For $x=1+\gamma \varpi^{j}$, we have

$$
\begin{aligned}
\chi^{w}(x) \chi(x)^{-1} & =\psi \circ \operatorname{Tr}_{k_{n} / k} \alpha_{1}\left(\delta \gamma^{\sigma^{s_{1}-j}}-\gamma \delta^{\sigma^{j}}\right)^{\sigma^{-s}} \\
& =\psi \circ \operatorname{Tr}_{k_{n} / k} \gamma\left(\delta^{\sigma^{j-s_{1}}} \alpha_{1}^{\sigma^{j}}-\delta^{\sigma^{j}} \alpha_{1}^{\sigma^{s_{1}}}\right) .
\end{aligned}
$$

Since $\chi^{w}(x) \chi(x)^{-1}=1$ by assumption, we must have

$$
\delta^{\sigma^{j-s_{1}}} \alpha_{1}^{\sigma^{j}}=\delta^{\sigma^{j}} \alpha_{1}^{\sigma^{s_{1}}}
$$

or

$$
\delta^{\sigma^{-s_{1}}} \alpha_{1}=\delta \alpha_{1}^{\sigma^{s_{1}-j}}
$$


or

$$
\left[\delta \varpi^{s_{1}-j}, \alpha_{1} \varpi^{-s}\right]=0 \text {. }
$$

From (9), $\alpha_{1} \varpi^{-s_{1}} \equiv \alpha_{0} \eta_{(j)}^{-s_{1} / f_{1}} \bmod P^{1-s_{1}}$, where $\alpha_{0}$ and $\eta_{(j)}^{-s_{1} / f_{1}}$ are central in $D_{(j)}$. Then $\left[\delta_{r} \eta_{(j)}^{r}, \alpha_{0} \eta_{(j)}^{-s_{1} / f}\right] \in P^{1-j}$, from the above calculation. But

$$
\begin{aligned}
{\left[\delta_{r} \eta_{(j)}^{r}, \alpha_{0} \eta_{(j)}^{-s_{1} / f_{1}}\right] } & =\delta_{r}\left[\eta_{(j)}^{r}, \alpha_{0} \eta_{(j)}^{-s_{1} / f_{1}}\right]+\left[\delta_{r}, \alpha_{0} \eta_{(j)}^{-s_{1} / f_{1}}\right] \eta_{(j)}^{r} \\
& =\left[\delta_{r}, \alpha_{0} \eta_{(j)}^{-s_{1} / f_{1}}\right] \eta_{(j)}^{r},
\end{aligned}
$$

and this last can be in $P^{1-j}$ only if $\delta_{r} \in k_{n / e_{1}}$. This proves property (1).

It will also be important to know that our choices of $\alpha_{2}$ and $\alpha_{2}^{\prime}$ have fixed a representative of $\chi$ in its orbit under conjugation by $D^{\times}$. Suppose that $\chi^{x}=$ $\chi^{\prime}=\chi_{0} \chi^{\#}$, where $\chi^{\#}(1+y)=\psi \circ \operatorname{Tr}_{D / F}\left(\beta_{2}^{\prime} \eta_{(j+1)}^{-j_{0}} y\right)$. Let $\beta_{2}=\operatorname{Tr}_{k_{n} / k_{n / e_{1}}} \beta_{2}^{\prime}$. Since $\chi^{x}=\chi$ on $K^{j+1}$, property (1) gives an expression for $\chi$. Taking conjugacy classes mod the subgroup of elements fixing $\chi$, we see that we may assume that $x=1+\delta \varpi^{s_{1}-j}$. But the computation performed when computing $\eta_{(j)}$ shows that for this $x, \chi^{x}(1+y)=\chi(1+y)$ when $y=\gamma \eta_{(j+1)}^{j_{0}}, \gamma \in k_{n / e_{1}}$. Therefore $\chi^{\sim}\left(\chi^{\prime}\right)^{-1}(1+y)=1$ for these $y$. Since

$$
\chi^{\sim}\left(\chi^{\prime}\right)^{-1}(1+y)=\psi \circ \operatorname{Tr}_{k_{n} / k_{n / e_{1}}}\left(\gamma^{\sigma^{-j}}\left(\alpha_{2}-\beta_{2}\right)\right)
$$

if $\gamma \in k_{n / e_{1}}, \alpha_{2}=\beta_{2}$. By our choice of $\alpha_{2}^{\prime}, \chi=\chi^{\#}$.

(b) Assume that $\alpha_{2} \eta_{(j+1)}^{j_{0}} \notin E_{(j+1)}$. Then $E_{(j+1)}\left[\alpha_{2} \eta_{(j+1)}^{-j_{0}}\right]$ is a field of ramification index $e_{2}$ and residue class degree $f_{2}$ over $F$, where $e_{2} f_{2}>e_{1} f_{1}$. We then let $S_{2}=j$. Note that (10) and (5) are now satisfied. It is also easy to check (6). Let $f_{2}=f_{2}^{\prime} f_{1}$; set $s_{1}-s_{2}=f_{1} r$. Note that $\eta_{(j+1)}=\eta_{1}$. Now suppose that $w$ commutes with $\chi$. Then $w$ commutes with $\left.\chi\right|_{K^{j+1}}$. From property (1).

$$
w=\delta_{0} \eta_{1}^{h}\left(1+\delta_{1} \eta+\cdots+\delta_{r} \eta_{1}^{r}+\delta_{s_{1}-j+1} \varpi^{s_{1}-j+1}+\cdots\right)=w_{1} w_{2},
$$

say. (Note the jump in indices between $r$ and $s_{1}-j+1$.) We show that $f_{2}^{\prime} \mid h$. Let $y=1+\gamma \eta_{1}^{j_{0}}=1+y_{1}$, with $\gamma \in k_{n / e_{1}}$. Then

$$
\chi\left(w y w^{-1} y^{-1}\right)=\chi_{0}\left(w y w^{-1} y^{-1}\right) \chi^{\sim}\left(w y w^{-1} y^{-1}\right) .
$$

By an application of Lemma 2.2 and Remark 2.3(b), we get $\chi_{0}\left(w y w^{-1} y^{-1}\right)=$ 1. Hence we need only consider $\chi^{\sim}$. But

$$
\begin{aligned}
\chi^{\sim}\left(w y w^{-1} y^{-1}\right) & =\psi \circ \operatorname{Tr}_{D / F} \alpha_{2}^{\prime} \eta_{1}^{-j_{0}}\left(w_{1} y_{1} w_{1}^{-1}-y_{1}\right) \\
& =\psi \circ \operatorname{Tr}_{k_{n} / k} \alpha_{2}^{\prime}\left[\delta_{0}\left(\delta_{0}^{-1}\right)^{\sigma_{2}} \gamma^{\sigma^{f_{1} h}}-\gamma\right]^{\sigma^{-s_{2}}} \\
& =\psi \circ \operatorname{Tr}_{k_{n / e_{1}} / k} \alpha_{2}\left[\delta_{0}\left(\delta_{0}^{-1}\right)^{\sigma_{2}} \gamma^{\sigma^{\delta_{1} h}}-\gamma\right]^{\sigma^{-s_{2}}}
\end{aligned}
$$

(all terms but $\alpha_{2}^{\prime}$ are in $k_{n / e_{1}}$ )

$$
=\psi \circ \operatorname{Tr}_{k_{n / e_{1}} / k} \gamma\left[\alpha_{2}^{\sigma^{s_{2}-f_{1} h}} \delta_{0}^{\sigma^{-f_{1} h}}\left(\delta_{0}^{-1}\right)^{\sigma^{s_{2}-f_{1} h}}-\alpha^{\sigma^{s_{2}}}\right]
$$


Since this must be 1 for all $\gamma \in k_{n / e_{1}}$, the coefficient of $\gamma$ must be 0 ; that is,

$$
\alpha_{2}^{\sigma^{s_{2}-f_{1} h}} \delta_{0}^{\sigma^{-f_{1} h}}=\alpha_{2} \delta_{0}^{\sigma^{s_{2}-f_{1} h}}, \quad \alpha_{2} \delta^{\sigma^{-s_{2}}}=\alpha_{2}^{\sigma^{f_{1} h}} \delta_{0}
$$

Thus $\left[\delta_{0} \eta_{1}^{h}, \alpha_{2} \eta_{1}^{-j_{0}}\right]=0$. Since $F_{f_{2}} \subseteq E_{(j+1)}\left[\alpha_{2} \eta_{1}^{-j_{0}}\right]$, therefore $\delta_{0} \eta_{1}^{h}$ commutes with $F_{f_{2}}$. Hence $f_{2} \mid h f_{1}$, or $f_{2}^{\prime} \mid h$. This argument also implies that $\delta_{0} \in k_{n / e_{2}}$ when $h=0$, since then we need $\left[\delta_{0}, \alpha_{2} \eta_{1}^{-j_{0}}\right]=0$.

Let $\beta \in k_{n}$ satisfy $\operatorname{Tr}_{k_{n} / k_{n / e_{1}}} \beta=1$. As in (a), we may assume that $\alpha_{2}^{\prime}=\alpha_{2} \beta$. We fix one element $\alpha_{2} \eta_{1}^{-j_{0}}$ in each conjugacy class under $k_{n / e_{1}}^{\times}\left\langle\eta_{1}\right\rangle$, and we may assume (conjugating by $\eta_{1}$ if necessary) that $\alpha_{2} \eta_{1}^{-j_{0}}$ is the representative of its conjugacy class. This fixes $\alpha_{2}^{\prime}$ and $\alpha_{2}$.

We now produce $\eta_{(j)}$, a generator of $P^{f_{2}}$, such that $\eta_{(j)}$ commutes with $\chi$, $\eta_{(j)}$ normalizes $k_{n / e_{2}}$, and $\eta_{(j)} \gamma \eta_{(j)}^{-1}=\gamma^{\sigma^{f_{2}}}$ for all $\gamma \in k_{n / e_{2}}$. We let $\eta_{(j)}=$ $\delta_{1} \eta_{1}^{f_{2}^{\prime}}\left(1+\delta_{0} \varpi^{t}\right)$, where $t=s_{1}-s_{2}$. We require that $\delta_{1} \in k_{n / e_{1}}$. Then $1+\delta_{0} \varpi^{t}$ commutes with $\left(\chi^{\sim}\right)^{\delta_{1} \eta_{1}^{f_{2}^{\prime}}}$ and $\delta_{1} \eta_{1}^{f_{2}}$ commutes with $\chi_{0}$, just as in (a). Also, for $y=1+\gamma \eta_{1}^{j_{0}}, \gamma \in k_{n / e_{1}}$, we have

$$
\begin{aligned}
\left(\chi^{\sim}\right)^{\delta_{1} \eta_{1}^{f_{2}^{\prime}}}(y) & =\psi \circ \operatorname{Tr}_{k_{n / e_{1}} / k}\left(\alpha_{2}\left[\left(\delta_{1} \gamma^{\sigma^{f_{2}}}\left(\delta_{1}^{\sigma^{s_{2}}}\right)^{-1}\right)^{\sigma^{-s_{2}}}-\gamma^{\sigma^{-s_{2}}}\right]\right) \\
& =\psi \circ \operatorname{Tr}_{k_{n / e_{1}} / k}\left(\gamma\left[\delta_{1}^{\sigma^{-f_{2}}}\left(\delta_{1}^{\sigma^{s_{2}-f_{2}}}\right)^{-1} \alpha_{2}^{\sigma_{2}^{s_{2}-f_{2}}}-\alpha_{2}^{\sigma_{2}^{s_{2}}}\right]\right) .
\end{aligned}
$$

This will be 1 for all such $\gamma$ if $\delta_{1}^{\sigma^{-f_{2}}} \alpha_{2}^{\sigma^{s_{2}-f_{2}}}=\alpha_{2}^{\sigma^{s_{2}}} \delta_{1}^{\sigma^{s_{2}-f_{2}}}$, or if $\delta_{1}^{\sigma^{-s_{2}}} \alpha_{2}=\alpha_{2}^{\sigma_{2}} \delta_{1}$, and this condition is met if we choose $\delta_{1}$ so that $\left[\delta_{1} \eta_{1}^{f_{2}^{\prime}}, \alpha_{2} \eta_{1}^{-j_{0}}\right]=0$. Since a prime element in $E_{(j+1)}\left[\alpha_{2} \eta_{1}^{-j_{0}}\right]$ has an $\left(n / e_{2} f_{2}\right)$ th root of the form $\delta_{1} \eta_{1}^{f_{2}^{\prime}}$, this choice is possible. (This determines $\delta_{1}$ only up to multiplication by an element of $k_{f_{2}}$, but the further choice of $\delta_{1}$ is arbitrary.) For this choice of $\delta_{1},(7)(\mathrm{i})$ guarantees a $\delta_{0}$ such that $\left(\chi^{\sim}\right)^{\delta_{1} \eta_{1}^{f_{2}^{\prime}}}\left(\chi^{\sim}\right)^{-1}=\left(\chi_{0}^{1+\delta_{0} \varpi^{\prime}} \chi_{0}^{-1}\right)^{-1}$, and for $\delta_{1}, \delta_{0}$ so chosen, we have $\chi^{\eta_{(j)}}=\chi$, just as in (a). The same argument as in (a) shows that $\eta_{(j)}$ conjugates $k_{n / e_{2}}$ appropriately. We note that, by a similar argument, $P^{g f_{2}}, g>0$, has a generator of the form $\delta_{1}^{\prime} \eta_{1}^{g f_{2}^{\prime}}\left(1+\delta_{0}^{\prime} \varpi^{t}\right)$ in $D_{2}$. This makes (8) clear, and (9) is also obvious.

We have verified (3). Recall that $D_{(j)}$ is generated by $k_{n / e_{2}}$ and $\eta_{(j)}$; set $E_{(j)}^{\sim}=F_{f_{2}}\left[\eta_{(j)}^{n / e_{2} f_{2}}\right]$. It is easy to see that $E_{(j)}^{\sim}$ is central in $D_{(j)}$ and that $e\left(E_{(j)}^{\sim} / F\right) \geq e_{2}$ and $f\left(E_{(j)}^{\sim} / F\right) \geq f_{2}$. Since $\left[D_{(j)}: F\right]=n^{2} / e_{2} f_{2}$, we must have $\left[E_{(j)}: F\right]=e_{2} f_{2}$. But $E_{(j)}^{\sim} \subseteq E_{(j)}$. Hence $E_{(j)}^{\sim}=E_{(j)}$, and (2) and (4) follow. For property $(1), D_{(j)}^{\times}$clearly commutes with $\chi$, and Lemma 2.2 shows that $D_{1}^{\times} \cap K^{1}$ and $K^{s_{1}-s_{2}+1}$ commute with $\chi$. For the converse, the inductive hypothesis and our calculation at the beginning of (b) (together with the same 
sort of argument used for (1) of (a)) show that if $w$ commutes with $\chi$, then $w=\delta_{0} \eta_{(j)}^{h}\left(1+\delta_{1}+\cdots+\delta_{t} \eta_{1}^{t}+\delta_{s_{1}-s_{2}+1} \varpi^{r+1}+\cdots\right)$, with $\delta_{0} \in k_{n / e_{2}}$, and $\delta_{l} \in k_{n / e_{1}}$ for $l<t$. We need only prove that $\delta_{r} \in k_{n / e_{1}}$, and this is done just as in (a).

Observe also that if $\chi^{\prime}$ is another extension of $\left.\chi\right|_{K^{j+1}}$ to $K^{j}$ constructed as above, then $\chi$ and $\chi^{\prime}$ are nonconjugate. Write $\chi^{\prime}=\chi_{0} \chi^{\#}$, where $\chi^{\#}(1+y)=$ $\psi \circ \operatorname{Tr}_{D / K}\left(\beta_{2}^{\prime} \eta_{1}^{\sigma^{-j_{0}}} y\right), \beta_{2}^{\prime} \in k_{n}$. For $y=\gamma \eta_{1}^{j_{0}}$ with $\gamma \in k_{n / e_{1}}, \chi^{\#}(1+y)=$ $\psi \circ \operatorname{Tr}_{k_{n / e_{1}} / k}\left(\beta_{2} \gamma^{\sigma^{-j_{0}}}\right), \beta_{2}=\operatorname{Tr}_{k_{n} / k_{n / e_{1}}}\left(\beta_{2}^{\prime}\right)$. Suppose that $\chi^{x}=\chi^{\prime}$. Since $\chi$ and $\chi^{\prime}$ agree on $K^{j+1}$, we can use (1) to get an expression for $\chi$. Ignoring terms known to commute with $\chi$, we may assume that $x=\delta \eta_{1}^{h}\left(1+\delta_{1}^{\prime} \varpi^{s_{2}-s_{1}}\right)$, $\delta \in k_{n / e_{1}}$. But a calculation done in verifying (7) shows that conjugation by $1+\delta_{1}^{\prime} \varpi^{s_{2}-s_{1}}$ fixes $\chi$ on elements $y=1+\gamma \eta_{1}^{j_{0}}, \gamma \in k_{n / e_{1}}$. Since $\delta \eta_{1}^{h}$ fixes $\chi_{0}$, we must have $\left(\chi^{\sim}\right)^{x_{0}}(y)=\chi^{\prime}(y)$ for these elements, where $x_{0}=\delta \eta_{1}^{h}$. A calculation shows that $x_{0}^{-1}\left(\alpha_{2} \eta_{1}^{-j_{0}}\right) x_{0}=\beta_{2} \eta_{1}^{-j_{0}}$. Since we chose one element from each conjugacy class, $\alpha_{2}=\beta_{2}$. From our other choices, $\alpha_{2}^{\prime}=\beta_{2}^{\prime}$ and $\chi=\chi^{\prime}$.

We still need (7). Write $j_{1}=h_{0} f_{1}$, and consider $\chi\left(w_{0} y w_{0}^{-1} y^{-1}\right)$, where $w_{0}=1+\delta_{1} \eta_{1}^{j_{0}-h_{0}}$ and $y=1+\gamma \eta_{1}^{h_{0}}\left(\delta_{1}, \gamma \in k_{n / e_{1}}\right)$. Some computation gives

$$
\chi\left(w_{0} y w_{0}^{-1} y^{-1}\right)=\psi \circ \operatorname{Tr}_{k_{n / e_{1}} / k} \gamma\left(\alpha_{2}^{\sigma^{j_{1}}} \delta_{1}^{\sigma^{j_{1}-s_{2}}}-\alpha_{2}^{\sigma^{s_{2}}} \delta^{\sigma^{j_{1}}}\right) .
$$

But

$$
\alpha_{2}^{\sigma^{j_{1}}} \delta_{1}^{\sigma^{j_{1} s_{2}}}-\alpha_{2}^{\sigma^{s_{2}}} \delta_{1}^{\sigma^{j_{1}}}=\left(\alpha_{2} \delta_{1}^{\sigma^{-s_{2}}}-\alpha_{2}^{\sigma^{s_{2}-j_{1}}} \delta_{1}\right)^{\sigma^{j_{1}}} \neq 0 \text { for } \delta_{1} \neq 0 \text { if } f_{2} \nmid j_{1} .
$$

Otherwise, $\alpha_{2} \eta_{1}^{-j_{0}}$ and $\delta_{1} \eta_{1}^{j_{0}-h_{0}}$ commute, and we have seen that this is impossible because of the calculation at the beginning of (b). Hence we can make an arbitrary character on the elements $y$ (as above) by choosing $\delta_{1}$ appropriately, and this proves (7)(ii). If $f_{2}^{\prime} \mid j_{1}$, the above character (3.11) is trivial when $\left[\alpha_{2} \eta_{1}^{-j_{0}}, \delta_{1} \eta_{1}^{j_{0}-h_{0}}\right]=0$, or whenever $\delta_{1} \eta_{1}^{j_{0}-h_{0}} \equiv \delta \eta_{(j)}^{\left(j_{0}-h_{0}\right) / f_{2}^{\prime}} \bmod P^{s_{2}-j_{1}+1}$ with $\delta \in k_{n / e_{2}}$. We then obtain only $\operatorname{Card}\left(k_{n / e_{1}} / k_{n / e_{2}}\right)$ distinct characters. But we also have

$$
\chi\left(w_{0} y w_{0}^{-1} y^{-1}\right)=\psi \circ \operatorname{Tr}_{k_{n / e_{1}} / k} \delta_{1}^{\sigma^{j_{1}-s_{1}}}\left(\gamma \alpha_{2}^{\sigma^{j_{1}}}-\gamma^{\sigma^{-s_{2}}} \alpha_{2}\right)
$$

If $\left[\gamma \eta_{1}^{h_{0}}, \alpha \eta_{1}^{-j_{0}}\right]=0$ for all $\gamma \in k_{n / e_{1}}$, this is always 1 on $y$. These are the $y$ with $\gamma \eta_{1}^{h_{0}} \equiv \gamma^{\prime} \eta_{(j)}^{h_{0} / f_{2}^{\prime}} \bmod P^{j_{0}+1}\left(\gamma^{\prime} \in k_{n / e_{2}}\right)$, by (10). Hence the characters we can obtain by varying $\delta_{1}$ are precisely the ones trivial on the elements $\gamma^{\prime} \eta_{2}^{h_{0} / f_{2}^{\prime}} \in D_{(j)}$. This is (7)(i).

(3.12) We now consider the general inductive step. We thus suppose that the hypotheses hold for $j+1$ and that we have defined the $s_{l}, e_{l}, f_{l}$ for $l \leq i$ (so that $\left.s_{i} \geq j+1\right)$. We now consider $\chi$ and $\chi_{0}$ on $K^{j}$. If $f_{i} \nmid j$, then (7)(ii) 
implies that we may assume after a conjugation that $\chi=\chi_{0}$. For this case, all the properties (2)-(10) are easy (most are vacuous), and (1) is straightforward (we will do a similar verification below). Thus we assume that $f_{i} \mid j$, and let $j=j_{0} f_{i}$. We have $\chi \cdot \chi_{0}^{-1}=\chi^{\sim}$ trivial on $K^{j+1}$ and $\chi^{\sim}\left(1+\gamma \eta_{(j+1)}^{j_{0}}\right)=\psi$ 。 $\operatorname{Tr}\left(\alpha_{j+1}^{\prime} \gamma^{\sigma^{j}}\right), \forall \gamma \in k_{n}$, for some element $\alpha_{i+1}^{\prime} \in k_{n}$. Let $\alpha_{i+1}=\operatorname{Tr}_{k_{n} / k_{n / e_{i}}} \alpha_{i+1}^{\prime}$. There are two cases, depending on whether $\alpha_{i+1} \eta_{(j+1)}^{j_{0}} \in E_{(j+1)}$ or not.

(3.13) (a) Assume first that $\alpha_{i+1} \eta_{(j+1)}^{j_{0}} \in E_{(j+1)}$. Then $\left(n / e_{i}\right) \mid j$, so that $\eta_{(j+1)}^{j_{0}}$ $\in E_{(j+1)}$; hence $\alpha_{i+1} \in k_{f_{i}}$. Let $\beta \in k_{n}$ satisfy $\operatorname{Tr}_{k_{n} / k_{n / f_{i}}} \beta=1$. By (7)(i) and an inductive argument, after conjugation we may assume that $\alpha_{i+1}^{\prime}=\beta \alpha_{i+1}$. (The induction uses (9) to show that the elements $1+\gamma_{l} \eta_{l}^{j / f_{l}}, \gamma_{l} \in k_{n / e_{l}}$, are congruent $\bmod K^{j+1}$ to elements $1+\gamma_{l-1} \eta_{l-1}^{j / f_{l-1}}$ with $\gamma_{l-1} \in k_{n / e_{l-1}}$.) In particular, if $\alpha_{j}=0$, then we may assume that $\alpha_{j}^{\prime}=0$ and that $\chi_{0}=\chi$. We shall show below that with this convention, different $\alpha_{j}$ give nonconjugate $\chi$.

We first need $\eta_{(j)}$. We define

$$
\eta_{(j)}=\eta_{(j+1)}\left(1+\delta_{i-1} \eta_{i-1}^{r_{i-1}}\right)\left(1+\delta_{i-2} \eta_{i-2}^{r_{i-2}}\right) \cdots\left(1+\delta_{0} \eta_{0}^{r_{0}}\right),
$$

where $r_{l} f_{l}=s_{l+1}-j, \eta_{0}=\varpi$, and the $\delta_{l} \in k_{n / e_{l}}$ are to be determined. We have, for $\gamma \in k_{n / e_{i}}$,

$$
\begin{aligned}
\left(\chi^{\eta_{(j+1)}^{-1}} \chi^{-1}\right)\left(1+\gamma \eta_{(j+1)}^{j_{0}}\right) & =\psi \circ \operatorname{Tr}_{k_{n} / k} \alpha_{i+1}^{\prime}\left(\gamma^{\sigma^{-f_{i}}}-\gamma\right) \\
& =\psi \circ \operatorname{Tr}_{k_{n / e_{i}} / k} \alpha_{i+1}\left(\gamma^{\sigma^{-f_{i}}}-\gamma\right) \\
& =\psi \circ \operatorname{Tr}_{k_{n / e_{i}} / k} \gamma\left(\alpha_{i+1} \gamma^{\sigma^{-f_{i}}}-\alpha_{i+1}\right)=1,
\end{aligned}
$$

since $\alpha \in k_{f_{i}}$. Thus, conjugation by $\eta_{(j+1)}$ fixes $\chi$ on $K^{j+1}$ and on elements $\left(1+\gamma \eta_{(j+1)}^{j_{0}}\right), \gamma \in k_{n / e_{i}}$. From (7)(i) and induction, we can choose the $\delta_{l}$ so that $\eta_{(j)}$ commutes with $\chi$. (As above, we use (9) in the inductive step.)

It is easy to check (as in (3.10)(a)) that $\eta_{(j)}$ satisfies (3) and (8). Set $\eta_{(j)}^{n / e_{i} f_{i}}=$ $\eta$. Then $\eta$ and $F_{f_{i}}$ are central in $D_{(j)}$, and $F_{f_{i}}[\eta]$ has ramification index over $F$ that is $\geq e_{i}$ (since $\eta$ generates $P^{n / e_{i}}$ ) and residue class degree $\geq f_{i}$ Since $\left[D_{(j)}: F\right]=n^{2} / e_{i} f_{i}$, the center $E_{(j)}$ of $D_{(j)}$ has degree $e_{i} f_{i}$ over $F$. Thus $F[\eta]=E_{(j)}$, and (2) and (4) follow. Property (9) is also obvious.

(3.14) Since (5)-(7) and (10) are essentially the same for $j$ and $j+1$, we need check only (1). It is straightforward (using Lemma 2.2 ) to verify that the elements of $K^{s_{1}-j+1}\left(K^{s_{2}-j+1} \cap D_{1}^{\times}\right) \cdots\left(K^{s_{i}-j+1} \cap D_{i-1}^{\times}\right) D_{(j)}^{\times}$commute with $\chi$. Conversely, suppose that $\chi^{w}=\chi$ for some $w$ in $D^{\times}$. Then $\chi^{w}=\chi$ on $K^{j+1}$; hence $w \in K^{s_{1}-j}\left(K^{s_{2}-j} \cap D_{1}^{\times}\right) \cdots\left(K^{s_{i}-j} \cap D_{i-1}^{\times}\right) D_{(j+1)}^{\times}$. Dividing by an element known to commute with $\chi$, we may assume (as in (3.10)(a)) 
that $w=1+\delta \eta_{i-1}^{\left(s_{i}-j\right) / f_{i-1}}+\cdots, \delta \in k_{n / e_{i-1}}$. (There are higher order terms of consequence. We deal with them later.) Our first task is to show that $w$ is congruent $\left(\bmod K^{s_{i}-j+1}\right)$ to an element in $D_{(j)}^{\times}$. Let $\gamma \in k_{n / e_{i-1}}$, and consider $\left(\chi^{w} \cdot \chi^{-1}\right)\left(1+\gamma \eta_{i-1}^{j_{0}}\right)$. Set $h_{0}=\left(s_{i}-j\right) / f_{i-1}$, and write $w=w_{1} w_{0}$, with $w_{0}=1+\delta \eta_{i-1}^{h_{0}}$. It is not hard to verify (using Lemma 2.2$)$ that $\chi^{w_{0}}\left(1+\gamma \eta_{i-1}^{j_{0}}\right)=$ $\chi\left(1+\gamma \eta_{i-1}^{j_{0}}\right)$ on these elements, since $\left(w_{0}, 1+\gamma \eta_{i-1}^{j_{0}}\right) \in K^{s_{i}+1}$ and the $\gamma \eta_{i-1}^{j_{0}}$ commute with $\left.\chi\right|_{K^{s_{i}+1}}$. Thus we are reduced to computing $\chi^{w_{1}} \chi^{-1}$ on these elements. Write $\chi=\chi_{0} \chi^{\sim}$ as in (3.12). Since $\chi_{0}^{w_{1}}=\chi_{0}$, we consider

$$
\begin{aligned}
\left(\chi^{\sim}\right)^{w_{1}}\left(\chi^{\sim}\right)^{-1}\left(1+\gamma \eta_{i-1}^{j_{0}}\right) & =\psi \circ \operatorname{Tr}_{k_{n / e_{i-1}} / k} \alpha_{i}\left(\delta \gamma^{\sigma^{s_{i}-j}}-\gamma \delta^{\sigma^{j}}\right)^{\sigma^{-s_{i}}} \\
& =\psi \circ \operatorname{Tr}_{k_{n / e_{i-1}} / k} \gamma\left(\delta^{\sigma^{j-s_{i}}} \alpha_{i}^{\sigma^{j}}-\delta^{\sigma^{j}} \alpha_{i}^{\sigma^{s_{i}}}\right) .
\end{aligned}
$$

(See (10) for the definition of $\alpha_{i}$.) This is to be 1 for all $\gamma \in k_{n / e_{i-1}}$. Then $0=$ $\left(\delta^{\sigma^{-s_{i}}} \alpha_{i-1} \delta \alpha_{i-1}^{\sigma_{i}^{s_{i} j}}\right)^{\sigma^{j}}$, or $\left[\delta \eta_{i-1}^{h_{0}}, \alpha_{i-1} \eta_{i-1}^{-j_{0}}\right]=0$. From (1), $\delta \eta_{i-1}^{h_{0}}$ is congruent $\bmod P^{s_{i}-j+1}$ to an element of $D_{i} ;(8)$ now implies that $\delta \eta_{i-1}^{h_{0}}$ is congruent $\bmod P^{s_{i}-j+1}$ to an element of $D_{(j)}$. This is what we need.

Using (8), we may also write

$w=1+\delta_{h_{0}} \eta_{(j)}^{h_{0}}+\delta_{h_{0}+1} \eta_{(j)}^{h_{0}+1}+\cdots+\delta_{h_{1}-1} \eta_{i-1}^{h_{1}-1}+\delta_{h_{1}} \eta_{i-2}^{h_{1}}+\cdots, \quad f_{i-2} h_{1}=s_{i-1}-j$, with the $\delta_{l} \in k_{n / e_{i-1}}$. Dividing by an element known to commute with $\chi$, we consider $w=1+\delta_{h_{1}} \eta_{i-2}^{h_{1}}+\cdots$, and an argument like the one above lets us show that $\delta_{h_{1}} \eta_{i-2}^{h_{1}} \in k_{n / e_{i-2}}$. Proceeding inductively, we get (1).

To see that different $\alpha_{i+1}$ give nonconjugate $\chi$, let $\chi_{1}=\chi_{0} \chi^{\#}$, where

$$
\chi^{\#}\left(1+\gamma \eta_{(j+1)}^{j_{0}}\right)=\psi \circ \operatorname{Tr}_{k_{n} / k}\left(\beta_{j+1}^{\prime} \gamma^{\sigma^{j}}\right), \quad \forall \gamma \in k_{n},
$$

and

$$
\alpha_{j+1} \neq \beta_{j+1}=\operatorname{Tr}_{k_{n} / k_{n / e_{i}}}\left(\beta_{j+1}^{\prime}\right) .
$$

If $\chi, \chi_{1}$ are conjugate by $x$, then $x$ fixes $\left.\chi\right|_{K^{j+1}}$. Therefore, $x$ is given by (1). Dividing by an element known to commute with $\chi$ (again by (1)), we have

$$
x=\delta \eta_{(j+1)}^{h}\left(1+\delta_{i-1} \eta_{i-1}^{r_{i}}\right) \cdots\left(1+\delta_{0} \varpi^{r_{1}}\right) .
$$

But we saw in constructing $\eta_{(j)}$ that for these $x, \chi^{x}(y)=\chi(y)$ for $y=$ $1+\gamma \eta_{(j)}^{j_{0}}, \gamma \in k_{n / e_{i}}$. Therefore $\chi^{\#}(y)=\chi^{\sim}(y)$ for these $y$. Since $\alpha_{i+1}, \beta_{i+1}$ determine $\chi^{\sim}, \chi^{\#}$, respectively on these elements, we must have $\alpha_{i+1}=\beta_{i+1}$. Therefore $\alpha_{i+1}^{\prime}=\beta_{i+1}^{\prime}$, as claimed.

(3.15) (b) Assume that $\alpha_{i+1} \eta_{(j+1)}^{j_{0}} \notin E_{(j+1)}$. Then $E_{(j+1)}\left[\alpha_{i+1} \eta_{(j+1)}^{j_{0}}\right]$ has ramification index $e_{i+1}$ and residue class degree $f_{i+1}$ over $F$, where $e_{i+1} f_{i+1}>e_{i} f_{i}$. This defines $e_{i+1}, f_{i+1}$. Let $\eta_{i}=\eta_{(j+1)}, s_{i+1}=j, s_{i}-s_{i+1}=f_{i} r, D_{i}=D_{(j+1)}$, 
$E_{i}=E_{(j+1)}$. Write $f_{i+1}=f_{i+1}^{\prime} f_{i}$. Properties (10) and (5) are now clear, and (6) is easy. We next show that if $w=\delta_{0} \eta_{i}^{h}\left(1+\delta_{1} \eta_{i}+\cdots\right)=w_{1} w_{2}$ commutes with $\chi$, then $f_{i+1}^{\prime} \mid h$. We may assume that $w$ commutes with $\left.\chi\right|_{K^{j+1}}$. Let $y=1+\gamma \eta_{i}^{j_{0}}=1+y_{1}$, with $\gamma \in k_{n / e_{i}}$. Then

$$
\chi\left(w y w^{-1} y^{-1}\right)=\chi_{0}\left(w y w^{-1} y^{-1}\right) \chi^{\sim}\left(w y w^{-1} y^{-1}\right) .
$$

However, a calculation using Lemma 2.2 (essentially the same as one in $3.10(\mathrm{~b})$ ) shows that $\chi_{0}\left(w y w^{-1} y^{-1}\right)=1$. The point is that $y_{1}$ commutes with $\chi_{0}$. We also have, by a computation that is by now standard,

$$
\chi^{\sim}\left(w y w^{-1} y^{-1}\right)=\psi \circ \operatorname{Tr}_{k_{n / e_{i}} / k} \gamma\left[\alpha_{i+1} \delta_{0}^{\sigma^{-s_{i+1}}}\left(\delta_{0}^{-1}\right)-\alpha_{i+1}^{\sigma^{f_{i} h}}\right]^{\sigma^{s_{i+1}}-f_{1} h} .
$$

Since this is to be 1 for all $\gamma \in k_{n / e_{i}}$, we must have $\alpha_{i+1} \delta_{0}^{\sigma^{-s_{2}}}=\delta_{0} \alpha_{i+1}^{\sigma^{f_{i} h}}$, or $\left[\alpha_{1+1} \eta_{i}^{-j_{0}}, \delta_{0} \eta_{i}^{h}\right]=0$. Since $E_{(j+1)}\left[\alpha_{i+1} \eta_{i}^{-j_{0}}\right] \supseteq F_{f_{i}}$ has residue class degree $f_{i+1}^{\prime}$ over $E_{(j+1)}$, we must have $f_{i+1}^{\prime} \mid h$. Similarly, we have $\delta_{0} \in k_{n / e_{i+1}}$ if $h=0$ $($ see $3.10(b))$.

Let $\beta \in k_{n}$ satisfy $\operatorname{Tr}_{k_{n} / k_{n / e_{i}}} \beta=1$. Then, as in (a), we may assume by using (7)(i) and (9) that $\alpha_{i+1}^{\prime}=\alpha_{i+1} \beta$. We fix one element $\alpha_{i+1} \eta_{i}^{-j_{0}}$ in each conjugacy class under $k_{n / e_{i}}\left\langle\eta_{i}\right\rangle$, and we may assume (conjugating if necessary) that $\alpha_{i+1}$ is that element of the conjugacy class. These choices fix $\alpha_{i+1}^{\prime}$ and $\alpha_{i+1}$. (That different $\alpha_{i+1}$ give nonconjugate $\chi$ is proved essentially as in (a); see the end of (3.14). We give some details below.)

(3.16) We next construct $\eta_{(j)}$. We set

$$
\eta_{(j)}=\delta_{i} \eta_{i}^{f_{i+1}^{\prime}}\left(1+\delta_{i-1} \eta_{i-1}^{r_{i-1}}\right)\left(1+\delta_{i-2} \eta_{i-2}^{r_{i-2}}\right) \cdots\left(1+\delta_{0} \eta_{0}^{r_{0}}\right),
$$

where $\eta_{0}=\varpi, r_{l} f_{l}=s_{l-1}-s_{i+1}$, and the $\delta_{l} \in k_{n / e_{l}}$ are to be determined. The construction is like that in (3.13). For $\gamma \in k_{n / e_{i}}$,

$$
\left(\chi^{\left(\delta_{i} \eta_{i}^{f_{i+1}^{\prime}}\right)^{-1}} \chi^{-1}\right)\left(1+\gamma \eta_{i}^{j_{0}}\right)=\psi \circ \operatorname{Tr}_{k_{n / e_{i}} / k} \gamma\left[\alpha_{i+1}^{\sigma_{i+1}^{f_{i+1}}} \delta_{i}\left(\delta_{i}^{\sigma^{-s_{i+1}}}\right)^{-1}-\alpha_{i+1}\right]^{\sigma^{-s_{i}}} .
$$

If we choose $\delta_{i}$ so that $\left[\delta_{i} \eta^{f_{i+1}^{\prime}}, \alpha_{i+1} \eta^{-s_{i}}\right]=0$, this will be 1 for all such $\gamma$. We can always choose such a $\delta_{i}$, since $\alpha_{i+1} \eta_{i}^{-s_{i}}$ generates a field of residue class $f_{i+1}^{\prime}$ in $D_{i}$. We next choose the $\delta_{l}$ inductively, using (7)(i) and (9). Now (9) for $j$ follows immediately. Because $\eta_{l}$ acts as $\sigma^{f_{l}}$ on $k_{n / e_{l}}$, it is easy to check that $\eta_{l}^{r_{l}}$ commutes with $k_{n / e_{i+1}}$; hence (3) holds. For (8), we can use the same argument to show that $P^{g f_{i+1}}$ has a generator in $D_{(j)}$ of the form $\delta_{i} \eta_{i}^{g f_{i+1}}\left(1+\delta_{i-1}^{\prime} \eta_{i-1}^{r_{i}}\right) \cdots\left(1+\delta_{0} \eta_{1}^{r_{1}}\right)$. This implies easily that if $x \in D_{i}^{\times} \cap K^{r}$, $r=g f_{i+1}$, then we can write $x\left(\bmod K^{r+s_{1}-s_{i}^{i}}\right)$ as

$$
x=x_{g} x_{g+1} \cdots x_{g+g_{l}}, \quad f_{l} g_{l}=s_{l}-s_{i},
$$


where $x_{g+l}=x_{g+1, i} \cdots x_{g+l, 0}, x_{g+l, b} \in D_{b}^{\times} \cap K^{r+l f_{i+1}+s_{b+1}-s_{i}}$. It is now not too hard to see that to verify (8) we need to show that if $y_{b} \in D_{b} \cap P^{r+s_{b+1}-s_{i}}$, $y_{c} \in D_{c} \cap P^{r+f_{i+1}+s_{c+1}-s_{i}}$, with $b<c$, then

$$
y_{b} y_{c} \in\left(D_{c} \cap P^{r+s_{c+1}-s_{i}}\right)+\left(D_{c-1} \cap P^{r+s_{c}-s_{i}}\right)+\cdots+P^{r+s_{1}-s_{i}} .
$$

Since

$$
y_{c} \in\left(D_{c+1} \cap P^{r+f_{i+1}+s_{c+1}-s_{i}}\right)+\left(D_{c-2} \cap P^{r+f_{i}+s_{c}-s_{i}}\right)+\cdots+P^{r+f_{i+1}+s_{1}-s_{i}},
$$

by (8) and the inductive hypothesis, we reduce the truth of the assertion with $b$ and $c$ to the truth for $b$ and $c-a, a>1$. An inductive argument now completes the proof of $(8)$.

It is clear that $\eta_{(j)}$ generates $P^{f_{i+1}}$ and that $\eta_{(j)}^{n / e_{i+1} f_{i+1}}$ is central in $D_{(j)}$. Let $E_{(j)}^{\sim}=F\left[\eta_{(j)}^{n / e_{i+1} f_{i+1}}\right]$. Then $E_{(j)}^{\sim}$ clearly has ramification index $\geq e_{i+1}$ and residue class degree $\geq f_{i+1}$ over $F$. On the other hand, $D_{(j)}$ is known to be of degree $\geq n^{2} / e_{i+1} f_{i+1}$ over $F$. Hence, its center, $E(j)$, has degree $e_{i+1} f_{i+1}$ over $F$. But $E(j) \supseteq E_{(j)}^{\sim}$. Thus, $E_{(j)}=E_{(j)}^{\sim}$. This proves (2) and (4).

(3.17) That leaves only properties (1) and (7). For (1), the verification that elements in $K^{s_{1}-j+1}\left(K^{s_{2}-j+1} \cap D_{1}^{\times}\right) \cdots D_{(j)}^{\times}$commute with $\chi$ is straightforward, using Lemma 2.2. Conversely, if $w$ commutes with $\chi$, then $w$ certainly commutes with $\left.\chi\right|_{K^{j+1}}$. Hence $w=\delta_{0} \eta_{i}^{h_{0}}\left(1+\delta_{1} \eta_{i}+\cdots\right), \delta_{0} \in k_{n / e_{i}}$. We say in (3.15) that $f_{i+1}^{\prime} \mid h_{0}$. Thus we may (possibly changing the $\delta_{i}$ ) rewrite $w=\eta_{(j)}^{h_{1}} \delta_{0}\left(1+\delta_{1} \eta_{1}+\cdots\right), h_{1} f_{i+1}^{\prime}=h_{0}$. Since $\eta_{(j)}^{h_{1}}$ commutes with $\chi$, we may assume that $h_{1}=0$. From (3.15) again, we must have $\delta_{0} \in k_{n / e_{i+1}} \subseteq D_{(j)}^{\times}$. Thus we may assume that $\delta_{0}=1$. The rest of the proof proceeds as in (a); see (3.14).

To see that the $\chi$ with distinct $\alpha_{i+1}^{\prime}$ are nonconjugate, let $\chi^{\prime}$ be another extension. If $\chi^{x}=\chi^{\prime}$, then $x$ fixes $\left.\chi\right|_{K^{j+1}}$. Comparing (1) for $j$ and $j+1$, we see that we may assume that $x=\delta_{i} \eta_{i}^{h}\left(1+\delta_{i-1} \eta_{i-1}^{r_{i-1}}\right) \cdots\left(1+\delta_{0} \eta_{0}^{r_{0}}\right), \delta_{l} \in$ $k_{n / e_{l}}$ and the $r_{l}$ as in (3.16). On elements $y=1+\gamma \eta_{i}^{j_{0}}$ with $\gamma \in k_{n / e_{i}}$, a calculation using Lemma 2.2 shows that $\chi^{x}(y)=\chi^{x_{0}}(y)$, where $x_{0}=\delta_{i} \eta_{i}^{h}$. We have $\chi=\chi_{0} \chi^{\sim}$, from (3.12). $x_{0}$ fixes $\chi_{0}$, and, for $y$ as above, $\left(\chi^{\sim}\right)^{x_{0}}(y)=$ $\psi \circ \operatorname{Tr}_{k_{n / e_{i}} / k}\left(\beta_{i+1} \gamma^{\sigma^{-j}}\right)$, where $\left(x_{0}\right)^{-1}\left(\alpha_{i+1} \eta_{i}^{-j_{0}}\right) x_{0}=\beta_{i+1} \eta_{i}^{-j_{0}}$. Since we fixed $\alpha_{i+1} \eta_{i}^{j_{0}}$ in this conjugacy class, $\alpha_{i+1}=\beta_{i+1}$. It follows that $\chi=\chi^{\prime}$.

(3.18) For (7), we may write $j_{0}=h_{0} f_{i}$. Let $w_{0}=1+\delta_{i} \eta_{i}^{j_{0}-h_{0}}$ and $y=1+\gamma \eta_{i}^{h_{0}}$ (where $\delta_{i}, \gamma \in k_{n / e_{i}}$ ). The standard computation (using Lemma 2.2 and the fact that $\delta_{i} \eta_{i}^{j_{0}-h_{0}}$ computes with $\left.\chi\right|_{K^{j+1}}$ ) gives

$$
\chi\left(w_{0} y w_{0}^{-1} y^{-1}\right)=\psi \circ \operatorname{Tr}_{k_{n / e_{i}} / k} \gamma\left(\alpha_{i+1} \delta_{1}^{\sigma^{-s_{i+1}}}-\alpha_{i+1}^{\sigma^{s_{i+1}-j_{1}}} \delta_{i}\right)^{\sigma^{j_{1}}} .
$$


If $f_{i+1}^{\prime} \nmid j_{1}$, then the second factor is nonzero for $\delta_{i} \neq 0$, since otherwise $\left[\alpha_{i+1} \eta_{i}^{-j_{0}}, \delta_{i} \eta_{i}^{h_{0}}\right]=0$, and this violates what was proved in (3.15). Hence we can, by choosing $w_{0}$ appropriately, conjugate to produce an arbitrary character on the element $y$ as above. This proves (7)(ii). If $f_{i+1}^{\prime} \mid j_{1}$, then (3.19) is trivial whenever $\delta_{i} \eta_{i}^{h_{0}} \equiv \delta \eta_{(j)}^{h_{0} / f_{i+1}^{\prime}}$ with $\delta \in k_{n / e_{i+1}}$, and we obtain exactly $\operatorname{Card}\left(k_{n / e_{i}} / k_{n / e_{i+1}}\right)$ distinct characters. But we can rewrite (3.19) as

$$
\chi\left(w_{0} y w_{0}^{-1} y^{-1}\right)=\psi \circ \operatorname{Tr}_{k_{n / e_{i}} / k} \delta_{i}^{\sigma^{j_{1}-s_{i+1}}}\left(\gamma \alpha_{i+1}^{\sigma^{j_{1}}}-\gamma^{\sigma^{-s_{i+1}}} \alpha_{i+1}\right),
$$

and this is always trivial for $\gamma \eta_{i}^{h_{0}} \equiv \gamma^{\prime} \eta_{(j)}^{h_{0} / f_{i+1}^{\prime}} \bmod P^{j_{0}+1}$ (with $\gamma^{\prime} \in k_{n / e_{i+1}}$ ), since then $\left[\gamma \eta_{i}^{h_{0}}, \alpha_{2} \eta_{i}^{-j_{0}}\right] \equiv 0 \bmod P^{j_{1}-s_{i+1}+1}$. Hence the characters we obtain by varying $\delta_{1}$ are precisely these that are trivial on the elements $1+\gamma^{\prime} \eta_{(j)}^{h_{0} / f_{i+1}^{\prime}} \in$ $D_{(j)}$, and (7)(i) follows.

And this completes the proof of Theorem 3.1.

Before continuing the construction, it may be helpful to pause and see where we are going.

Let $\chi$ be as in Theorem 3.1, and define $s_{i}^{\prime}=\left[s_{i} / 2\right]+1, s_{i}^{\prime \prime}=s_{i}+1-s_{i}^{\prime}$, in analogy with $s_{1}^{\prime}, s_{1}^{\prime \prime}$. We would like to construct all the irreducible unitary representations of $D^{\times}$containing $\chi$. Mackey theory says that we do this by considering the group $H_{0}$ of elements $x$ with $\chi^{x}=\chi$, constructing all irreducibles $\rho$ of $H_{0}$ such that $\rho$ contains $\chi$, and then inducing to $D^{\times}$. Theorem 3.1 even tells us what $H_{0}$ is. Unfortunately, it is not obvious how to construct the desired representations of $H_{0}$. There is one special case, however, where things are not so bad. If $s_{r_{1}}=1$, then $H_{0}=K^{s_{1}^{\prime \prime}} D_{1}^{\times}$. We can extend $\chi$ to $K^{s_{1}^{\prime}} D_{1}^{\times}$(this is proved in Lemma 4.2), and the further extension to $H_{0}$ is not hard. That gives us one representation of $H_{0}$ containing $\chi$, and the rest are obtained by tensoring that one with representations of $D_{1}^{\times}$trivial on $D_{1}^{\times} \cap K^{s_{1}^{\prime}}$. Since the dimension of $D_{1}$ over its center is $<n^{2}$, we may assume inductively that we know these representations.

In general, however, we need to continue the construction. Suppose that $r_{1} \geq 2$. If we extend $\chi$ to $K^{s_{1}^{\prime}}\left(D_{1}^{\times} \cap K^{s_{1}^{\prime}-1}\right)$, the elements of $D_{1}^{\times} \cap K^{s_{2}-s_{1}^{\prime}}$ will in general not commute with $\chi$ (just as elements of $K^{1}$ did not generally commute with $\left.\left.\chi\right|_{K^{s_{1}-1}}\right)$. This suggests that we follow the procedure of Theorem 3.1 and extend $\chi$ to $K^{s_{1}^{\prime}}\left(D_{1}^{\times} \cap K^{s_{2}^{\prime}}\right)$. With luck, the group of elements commuting with $\chi$ will be $K^{s_{1}^{\prime \prime}}\left(K^{s_{2}^{\prime \prime}} \cap D_{1}^{\times}\right)\left(K^{s_{3}-s_{2}^{\prime}} \cap D_{2}^{\times}\right) \cdots\left(K^{s_{r_{1}-1}-s^{\prime}} \cap D_{r_{1}-1}^{\times}\right) D_{r_{1}}^{\times}$, and we will have made progress. In particular, we will be in a position to complete the construction as above when $r_{1}=2$. If $r_{1}>2$, then we can repeat the last step, and so on. The problem is that the procedure of Theorem 3.1 may introduce 
more triples $\left(s_{i}, e_{i}, f_{i}\right)$ with $i>r_{1}$. Thus, even after $r_{1}$ iterations we may have to continue further. Luckily, the procedure must be finite because $n$ is finite.

This discussion may help to motivate the next definition. We say that a pair $\left(\chi^{\prime}, H\right)$, where $H$ is a subgroup of $D^{\times}$and $\chi^{\prime}$ is a character on $H$, is satisfactory if there are triples $\left(s_{i}, e_{i}, f_{i}\right)$ and elements $\eta_{i}, 1 \leq i \leq r_{0}$, such that

(a) $H=K^{s_{1}^{\prime}}\left(K^{s_{2}^{\prime}} \cap D_{1}^{\times}\right) \cdots\left(K^{s_{r_{0}}^{\prime}} \cap D_{r_{0}-1}^{\times}\right)$, where $D_{i}$ is the division algebra generated by $\eta_{i}$ and $k_{n / e_{i}}$;

(b) the group of elements in $D^{\times}$commuting with $\chi^{\prime}$ is $K^{s_{1}^{\prime \prime}}\left(K^{s_{2}^{\prime \prime}} \cap D_{1}^{\times}\right) \cdots$ $\left(K^{s_{r_{0}}^{\prime \prime}} \cap D_{r_{0}-1}^{\times}\right) D_{r_{0}}^{\times}=H^{\sim}$ (the numbers $s_{i}^{\prime}, s_{1}^{\prime \prime}$ were defined above);

(c) there are elements $\eta_{(j)}$ as in Theorem 3.1, with $\eta_{i}=\eta_{\left(s_{i+1}+1\right)}$ and $\eta_{r_{0}}=$ $\eta_{\left(s_{r_{0}}^{\prime}\right)}$, such that properties (1)-(10) of Theorem 3.1 (see also (3.9)) hold.

Note that $H^{\sim}$ normalizes $H$.

\section{(4.1) Proposition. The character $\chi$ of Theorem 3.1 has a satisfactory extension.}

Proof. The proof will in fact show that $\chi$ has "sufficiently many" satisfactory extensions, in a sense to be made precise as we continue.

Let $\chi, r_{1}$ be as in Theorem 3.1. If $r_{1}=1$, then we observed above that $\left(K^{s_{1}^{\prime}}, \chi\right)$ is already satisfactory. So we may assume that $r_{1}>1$. In that case, consider the group $H_{1}=K^{s_{1}^{\prime}}\left(K^{s_{2}^{\prime}} \cap D_{1}^{\times}\right)$. Clearly $\left(H_{1}, H_{1}\right) \subseteq K^{s_{1}^{\prime}}$, since $2 s_{2}^{\prime} \geq$ $s_{2}>s_{1}^{\prime}$. Furthermore, $\chi \equiv 1$ on $\left(H_{1}, H_{1}\right)$. To verify this, it suffices to calculate $\chi((u, v))$ when each of $u, v$ is in either $K^{s_{1}^{\prime}}$ or $K^{s_{2}^{\prime}} \cap D_{1}^{\times}$. If $v$, say, is in $K^{s_{1}^{\prime}}$, then $\chi((u, v))=1$ because $u$ commutes with $\chi$. If $u, v \in K^{s_{2}^{\prime}} \cap D_{1}^{\times}$, then $\chi(u, v)=1$ by an application of Lemma 2.2. (Write $u=1+u_{0}$. Then $u_{0}$ commutes with $\left.\chi\right|_{K^{s_{2}}}$.) It follows that $\chi$ has an extension to $H_{1}$. Furthermore, $H_{1} / K^{s_{1}^{\prime}}$ is Abelian, so that $\chi$ has many such extensions. Choose one, which we also call $\chi$.

We now apply the procedure of Theorem 3.1 to $\chi$. While a complete account of what happens would be tedious, it may help to see the first step or so. We first extend $\left.\chi\right|_{K_{1}^{s_{1}^{\prime}}}$ to $\chi_{0}$ on $H_{1} \cap K^{s_{1}^{\prime}-1}$ by using Lemma 3.8 (the proof applies without change). Let $j=s_{1}^{\prime}-1, i=r_{1}$. Now consider our extension $\chi$ to $H_{1} \cap K^{s_{1}^{\prime}-1}$. If $f_{r_{1}}+s_{1}^{\prime}-1$, then $\chi$ and $\chi_{0}$ are conjugate, by (7)(ii) (the argument is that of (3.12)), and we may take $\chi=\chi_{0}$. We can then show, as before, that if one sets $\eta_{(j)}=\eta_{(j+1)}, D_{(j)}=D_{(j+1)}$, etc., then we have the analogue of property (1) of Theorem 3.1 :

(1)

$$
\begin{aligned}
\chi^{x}=\chi & \text { on } \bigcap H_{1} \cap K^{s_{1}^{\prime}-1} \cap x^{-1}\left(H_{1} \cap K^{s_{1}^{\prime}-1}\right) x \\
& \Leftrightarrow x \in K^{s_{1}^{\prime \prime}}\left(K^{s_{1}-j+1} \cap D_{1}^{\times}\right) \cdots\left(K^{s_{i}-j+1} \cap D_{i-1}^{\times}\right) D_{(j)}^{\times},
\end{aligned}
$$


and properties (2)-(10) of Theorem 3.1 also hold. If $f_{i} \mid j$, then write

$$
\begin{gathered}
\chi=\chi_{0} \chi^{\sim}, \\
\chi^{\sim}\left(1+\gamma \eta_{(j+1)}^{j_{0}}\right)=\psi \circ \operatorname{Tr}_{k_{n / e_{1}} / k}\left(\alpha^{\prime} \gamma^{\sigma^{-j}}\right), \quad \alpha^{\prime} \in k_{n / e_{1}}, j_{0} f_{i}=j .
\end{gathered}
$$

Let $\alpha=\operatorname{Tr}_{k_{n / e_{1}} / k_{n / e_{i}}} \alpha^{\prime}$. If $\alpha \eta_{(j+1)}^{-j_{0}} \in E_{(j+1)}$, then we use the arguments of (3.13) and (3.14) to produce $\eta_{(j)}$ (generating $\left.P^{f_{i}}\right), E_{(j)}$, and $D_{(j)}$ so that (1)-(10) of Theorem 3.1 hold. (1) takes the modified form given above. If $E_{(j+1)}\left[\alpha \eta_{(j+1)}^{-j_{0}}\right]$ is a proper extension of $E_{(j+1)}$ with ramification index $e_{i+1}$ and residue class degree $f_{i+1}$ over $F$, then set $s_{i+1}=j$, so that $\left(s_{i+1}, e_{i+1}, f_{i+1}\right)$ is the next triple for $\chi$. Let $\eta_{(j+1)}=\eta_{i}, D_{(j+1)}=D_{i}$, etc. Now the argument of (3.15)(3.18) produces $\eta_{(j)}, D_{(j)}$, etc., satisfying (1)-(10) of Theorem 3.1. Continue this procedure.

In this way, we obtain further triples $\left(s_{i}, e_{i}, f_{i}\right), r_{1}<i \leq r_{2}$ (say), together with elements $\eta_{(j)}$, division algebras $D_{(j)}$, and fields $E_{(j)}, j \geq s_{2}^{\prime}$, such that (1)-(10) of Theorem 3.1 hold. If $r_{2}=2$ (this happens when $r_{1}=2$ and we were never in case (b) of the proof of Theorem 3.1 for $j<s_{1}$ ), then $\left(H_{1}, \chi\right)$ is satisfactory $\left((1)\right.$ then says that $\chi^{x}=\chi$ on $H_{1} \cap x^{-1} H_{1} x$ iff $x \in$ $\left.K^{s_{1}^{\prime \prime}}\left(K^{s_{2}^{\prime \prime}} \cap D_{1}^{\times}\right) D_{2}^{\times}\right)$. If not, let $H_{2}=H_{1}\left(K^{s_{3}^{\prime \prime}} \cap D_{2}^{\times}\right)$. As above, $\left(H_{2}, H_{2}\right) \subseteq H_{2}$ and $\chi \equiv 1$ on $\left(H_{2}, H_{2}\right)$; the reasoning is virtually the same. We then apply the procedure to the extensions of $\chi$ to $H_{2}$.

Continue inductively. The procedure stops with a satisfactory $\left(\chi, H_{i-1}\right)$ if $s_{r_{i}}=i$. This is bound to happen for one of two reasons. If $s_{1}$ is large, we use the fact that $s_{r_{i}} \leq s_{1}\left(2^{1-r_{i}}\right)$ and $r_{i} \leq$ the number of prime divisors (with multiplicities) of $n$. In any case, $s_{r_{i}} \geq 2$ if $s_{1} \geq 2$, and this will stop the procedure.

(4.2) Lemma. With notation as above, let $\left(\chi^{\prime}, H\right)$ be satisfactory. Then $\chi^{\prime}$ extends as a character to $H \cdot D_{r_{0}}^{\times}$.

Proof. We need to show that if $u_{j}, v_{j} \in H \cdot D_{r_{0}}^{\times}$and $w=\prod_{j=1}^{g}\left(u_{j}, v_{j}\right) \in H$, then $\chi(w)=1$. By appropriate manipulations (described, e.g., in [4] and [5]), we may assume that each $u_{j}, v_{j}$ is in either $H$ or $D_{r_{0}}^{\times}$and that the terms with one of $u_{j}, v_{j} \in H$ come first in the product. If, say, $u_{1} \in H$, then $\chi^{\prime}\left(\left(u_{1}, v_{1}\right)\right)=1$ because $v_{1} \in H^{\sim}$ commutes with $\chi^{\prime}$. Thus we may assume without loss of generality that all $u_{j}, v_{j} \in D_{r_{0}}^{\times}$. Hence $w \in\left(D_{r_{0}}^{\times}, D_{r_{0}}^{\times}\right) \cap H$. By Lemma $2.1, w$ is $\left(\bmod K^{s_{1}+1}\right)$ a product of commutators $\left(u_{j}^{\prime}, v_{j}^{\prime}\right)$ with each $u_{j} \in D_{j}^{r_{0}} \subseteq H^{\sim}, v_{j}^{\prime} \in D_{r}^{\times} \cap K^{s_{r}^{\prime}} \subseteq H$. (This last comes from Theorem 3.1(8).) Therefore $\chi^{\prime}(w)=1$.

Fix an extension of $\chi^{\prime}$ to $H \cdot D_{r_{0}}^{\times}$, which we also call $\chi^{\prime}$. We now produce other representations of $D_{r_{0}}^{\times} H$ by tensoring with any irreducible representation 
$\rho$ of $D_{r_{0}}^{\times}$trivial on $D_{r_{0}}^{\times} \cap K^{s_{i}^{\prime}}$. We then produce a representation $\tau$ of $H^{\sim}$ by tensoring with Weil representations. This step is described in detail in [12, 9, and 1]. Here is a brief sketch. Suppose that $s_{1}$ is even, so that $s_{1}^{\prime \prime}=s_{1}^{\prime}-1$. There is a unique irreducible projective representation $\lambda_{1}$ of $K^{s_{1}^{\prime \prime}}$ that is trivial on $K^{s_{1}^{\prime}}$ and satisfies

$$
\lambda_{1}\left(x_{1}\right) \lambda_{1}\left(x_{2}\right) \lambda_{1}\left(x_{1}^{-1}\right) \lambda_{1}\left(x_{2}^{-1}\right)=\chi\left(x_{1} x_{2} x_{1}^{-1} x_{2}^{-1}\right) I
$$

for $x_{j}=1+\gamma_{j} \omega^{s_{1}^{\prime \prime}}$. Then $\left(\operatorname{dim} \lambda_{1}\right)^{2}=\left[K^{s_{1}^{\prime \prime}}: K^{s_{1}^{\prime}}\left(H \cap K^{s_{1}^{\prime \prime}}\right)\right]$, and $\lambda_{1}$ extends canonically to a projective representation $W_{1}$ of $H D_{r_{0}}^{\times} K^{s_{1}^{\prime \prime}}$. When $p$ is odd, this is the Weil (or oscillator) representation. For $p=2$, a description is given in Chapter 1 of [9], and a different (but equivalent) description for general $p$ is found in $\S 8$ of [1]. It turns out that $W$ is trivial on $K^{1} \cap H D_{r_{0}}^{\times}$. We now tensor $W$ with $\chi \otimes \rho$ to get an ordinary irreducible representation on $H D_{r_{0}}^{\times} K^{s_{1}^{\prime \prime}}$. If all $s_{j}$ with $j>1$ are odd, this representation is $\tau$. If not, we repeat this construction for each even $s_{j}, j>1$, to get $\tau$.

(4.3) Theorem. (a) For $\tau$ as above, $\operatorname{Ind}_{H^{\sim}}^{G} \tau=\pi$ is irreducible.

(b) Let $\tau_{1}, \tau_{2}$ (on $H_{1}^{\sim}, H_{2}^{\sim}$ ) be constructed as above, from $\chi_{1}^{\prime} \otimes \rho_{1}, \chi_{2}^{\prime} \otimes \rho_{2}$. Suppose that $\pi_{1} \cong \pi_{2}$. Then $H_{1}^{\sim}=H_{2}^{\sim}, \chi_{1}^{\prime}=\chi_{2}^{\prime}$, and $\rho_{1} \cong \rho_{2}$.

Proof. For (a), write $\tau^{w}(x)=\tau\left(w x w^{-1}\right)$. If $\tau^{w}$ and $\tau$ intertwine on their common domain, then their restrictions to the normal subgroup $K^{s_{1}^{\prime}}$ intertwine. Since $\left.\tau\right|_{K_{1}^{s_{1}^{\prime}}}$ is a multiple of $\chi,\left.\tau^{w}\right|_{K_{1}^{s_{1}^{\prime}}}$ is a multiple of $\chi^{w}$, and thus $\chi^{w}=\chi$. Therefore, $w \in K^{s_{1}^{\prime \prime}}\left(D_{1}^{\times} \cap K^{s_{2}-s_{1}^{\prime}}\right) \cdots\left(D_{r_{1}-1}^{\times} \cap K^{s_{r_{1}}-1-s_{1}^{\prime}}\right) D_{\left(s_{1}^{\prime}\right)}^{\times}$, by Theorem 3.1. This group normalizes $K^{s_{1}^{\prime}}\left(D_{1}^{\times} \cap K^{s_{2}^{\prime}}\right)$, and hence $\tau$ and $\tau^{w}$ intertwine when restricted to this group. Again, $\tau$ is a multiple of $\chi$ and $\tau^{w}$ is a multiple of $\chi^{w}$. Hence, $w \in K^{s_{1}^{\prime \prime}}\left(D_{1}^{\times} \cap K^{s_{2}^{\prime \prime}}\right)\left(D_{2}^{\times} \cap K^{s_{3}-s_{2}^{\prime}}\right) \cdots\left(D_{r_{2}-1}^{\times} \cap K^{s_{r_{2}-1}-s_{2}^{\prime}}\right) D_{\left(s_{2}^{\prime}\right)}^{\times}$. Proceeding inductively, we get $w \in H^{\sim}$. By a standard theorem ([16]), this proves irreducibility.

For (b), assume that there is $w \in D^{\times}$so that $\tau_{1}$ and $\tau_{2}^{w}$ intertwine. Then their restrictions to $K^{s_{1}^{\prime}}$ intertwine, and these are multiples of $\chi_{1}, \chi_{2}^{w}$, respectively. Therefore $\chi_{1}, \chi_{2}$ are conjugate under $D^{\times}$. From the uniqueness of the $\alpha_{i+1}^{\prime}$ in (3.12), this means that $\chi_{1}=\chi_{2}$ on $K^{s_{1}^{\prime}}$. From Theorem 3.1,

$$
w \in K^{s_{1}^{\prime \prime}}\left(D_{1}^{\times} \cap K^{s_{2}-s_{1}^{\prime}}\right) \cdots\left(D_{\left(s_{1}^{\prime}\right)}^{\times}\right) .
$$

As above, this means that $\chi_{1}, \chi_{2}$ are conjugate on $K^{s_{1}^{\prime}}\left(D_{1}^{\times} \cap K^{s_{2}^{\prime}}\right)$. By our construction, $\chi_{1}=\chi_{2}$ on $K^{s_{1}^{\prime}}\left(D_{1}^{\times} \cap K^{s_{2}^{\prime}}\right)$. By induction, we get $\chi_{1}=\chi_{2}$ and $w \in H_{1}^{\sim}=H_{2}^{\sim}$. But this immediately gives $\rho_{1} \cong \rho_{2}$.

(4.4) Remark. We could also prove (b) by another argument. It is evident from Theorem 3.1 and Proposition 4.1 that we have considered every completely 
satisfactory $\chi$ and every representation containing such a $\chi$. Thus, we have certainly constructed all of $\left(D^{\times}\right)^{\wedge}$, modulo tensoring with characters. We will see in the next section that the number of representations with kernel containing $K^{m+1}\left\langle\varpi^{n}\right\rangle$ is precisely the number of such representations we have constructed. Therefore, those constructed above must be distinct.

\section{5}

If we make the inductive assumption that every irreducible representation of division algebras of index $<n$ are constructed as in $\S \S 3$ and 4 , then the representation $\rho$ of $D_{r}^{\times}$in $\S 4$ has associated to it a sequence of triples $\left(s_{1}^{*}, e_{1}^{*}, f_{1}^{*}\right)$, $\ldots,\left(s_{t}^{*}, e_{t}^{*}, f_{t}^{*}\right)$. (This sequence is empty if $\rho$ factors through the norm map.) If $\left(s_{1}, e_{1}, f_{1}\right), \ldots,\left(s_{r_{0}}, e_{r_{0}}, f_{r_{0}}\right)$ is the sequence associated to $\chi$, we associate to $\rho$ the sequence

$$
\left(s_{1}, e_{1}, f_{1}\right), \ldots,\left(s_{r_{0}}, e_{r_{0}}, f_{r_{0}}\right),\left(s_{r_{0}+1}, e_{r_{0}+1}, f_{r_{0}+1}\right), \ldots,\left(s_{r_{0}+t}, e_{r_{0}+t}, f_{r_{0}+t}\right),
$$

where $s_{r_{0}+j}=s_{j}^{*} f_{r_{0}}, e_{r_{0}+j}=e_{j}^{*} e_{r_{0}}$, and $f_{r_{0}+j}=f_{j}^{*} f_{r_{0}}$. Notice that $\rho$ depends on the choice of extension of $\chi^{\prime}$ to $D_{r_{0}}^{\times}$, but that this choice does not affect the sequence of triples because two representations that differ by a 1-dimensional representation generate the same sequence. We shall say that $\pi$ is of type $\left\{\left(s_{1}, e_{1}, f_{1}\right), \ldots,\left(s_{r}, e_{r}, f_{r}\right)\right\}, r=r_{0}+t$. Note that $\pi$ is of type $\phi$ iff it is 1dimensional. The conductor of $\pi$ is, as usual, the smallest integer $m+1$ such that $\left.\pi\right|_{K^{m+1}}$ is trivial. If $\pi$ is trivial on $K$, the conductor will be 0 . We also say that $\pi$ has ramification index $e_{r}$ and residue class degree $f_{r}$, and write $e(\pi)=e_{r}, f(\pi)=f_{r}$. If $\pi$ is of type $\phi$, we define $e(\pi)=f(\pi)=1$.

Observe that our construction of representations of $D^{\times}$applies also to $K$. The one difference is that in those cases where we conjugated by $\varpi$ or $\eta_{(j)}$ to specify an element in the conjugacy class of $\chi$ (e.g., immediately before Theorem 3.1, or in (3.11)), we can no longer perform this conjugation. Thus we obtain more conjugacy classes.

We use this observation to prove

(5.1) Theorem. The number of representations $\pi$ of $D^{\times}$(constructed by tensoring those of Theorem 4.1 with characters) that satisfy

(a) $\pi\left(\varpi^{n}\right)=I$,

(b) $f(\pi) \mid f$, where $f$ is a fixed divisor of $n$,

(c) the conductor of $\pi$ is $\leq m+1$,

is

$$
A_{n ; m ; f}=\frac{n}{f^{2}} \sum_{d \mid f} \mu\left(\frac{f}{d}\right)\left(q^{d}-1\right) q^{d[m / f]},
$$

where $\mu=$ Möbius function and [ ] is the greatest integer function.

Proof. We follow the reasoning used in $\S 11$ of [13]. Let $g f=n$. We consider representations $\lambda$ of $K$ with $e(\lambda) \leq g, f(\lambda) \leq f$, and conductor $\leq m+1$. Consider the construction of $\chi$. We must have $\frac{n}{g} \mid s_{i}$, since otherwise $e(\lambda)>g$. 
Similarly, $\chi_{0}=\chi$ at each level $j$ where $\frac{n}{g} \nmid j$. When $\frac{n}{g} \mid j$, we do not need to have $\chi_{0}=\chi$, but the element $\alpha_{i+1} \eta_{(j)}$ (of (3.9)) must generate a field whose residue class degree is $\leq f$. Since $\alpha_{i+1}$ could be 0 , we see that there are $q^{f}$ different choices for $\alpha_{i+1}$ at each $j$ with $f \mid j$ and $0<j \leq m$. That gives $q^{f[m g / n]}$ choices (again, [ ] is the greatest integer function), and there are another $q^{f}-1$ choices at $K / K^{1}$. Hence the total number of representations of $K$ satisfying the conditions is $q^{f[m g / n]}\left(q^{f}-1\right)$. Möbius inversion now gives the number of representations $\lambda$ with $f(\lambda)=f, e(\lambda) \leq g$, and conductor $\leq m+1$ to be

$$
N_{n, m, f}=\sum_{d \mid f} \mu(f / d)\left(q^{d}-1\right) q^{d[m / f]} .
$$

Each such $\lambda$ has $f$ inequivalent conjugates under $D^{\times}$, and the group of elements in $D^{\times}$fixing $\lambda$ has degree $\frac{n}{f}$ over $K$. Hence there are $\frac{n}{f^{2}} N_{n, m, f}$ representations of $\pi$ meeting the conditions of the theorem.

(5.2) Corollary. The total number of irreducible unitary representations of $D^{\times}$ with conductor $\leq m+1$ that are trivial on $\varpi^{n}$ and are constructed as in $\S \S 3$ and 4 is

$$
A_{n ; m}=\sum_{f \mid n} \sum_{d \mid f} \frac{n}{f^{2}} \mu(f / d)\left(q^{d}-1\right) q^{d[m / f]} .
$$

(5.3) Corollary. Every irreducible unitary representation of $D^{\times}$is constructed as in $\S \S 3$ and 4.

Proof. It suffices to consider irreducible representations $\pi$ trivial on $\varpi$. From the main theorem of [13], the number of conjugacy classes of $D^{\times} / K^{m+1}\langle\varpi\rangle$ is $A_{n ; m}$. This, of course, extends the inductive assumption made at the beginning of this section.

(5.4) Corollary. The number of irreducible unitary representations $\pi$ of $D^{\times}$ trivial on $\left\langle\varpi^{n}\right\rangle$ with ramification index $\frac{n}{f}$, residue class degree $f$, and conductor $\leq m$ is

$$
B_{n ; m ; f}=\sum_{d \mid f} \sum_{c \mid(n / f)} \frac{n}{c f^{2}} \mu\left(\frac{n}{c f}\right) \mu\left(\frac{f}{d}\right)\left(q^{d}-1\right) q^{d[m / f]} .
$$

Proof. If $\pi$ has ramification index $e$ and residue class degree $f$, with $e f \mid n$, then $\pi$ corresponds to a representation $\pi^{\sim}$ of $D_{e f}^{\times}$. Thus, we obtain $B_{n ; m ; f}$ by Möbius inversion from Theorem 5.1.

Define an element $\pi$ of $\left(D_{n}^{\times}\right)^{\wedge}$ to be basic if its conductor (the smallest number $s+1$ such that $\left.K^{s+1} \subseteq \operatorname{Ker} \pi\right)$ cannot be reduced by twisting with a character (if $K \subset \operatorname{Ker} \pi$, then we require $\pi$ to be trivial). Except for the trivial representation, these are the irreducibles constructed in the previous sections. Of course, every element of $\left(D_{n}^{\times}\right)^{\wedge}$ is the tensor product of a basic representation with a 1-dimensional representation, and it is also easy to determine how many basic representations are obtained from a given one by twisting 
with a character. The next theorem gives a parametrization of sorts for basic representations.

(5.5) Theorem. Let $\left(s_{1}, e_{1}, f_{1}\right), \ldots,\left(s_{r}, e_{r}, f_{r}\right)$ be a sequence of triples satisfying

(i) $s_{i}>s_{2}>\cdots s_{r} \geq 0$,

(ii) $e_{1}\left|e_{2}\right| \cdots\left|e_{r}, f_{1}\right| f_{2}|\cdots| f_{r}$, and $1<e_{1} f_{1}<e_{2} f_{2}<\cdots<e_{r} f_{r} \leq n$,

(iii) $e_{r} f_{r} \mid n$,

(iv) $f_{r} \mid s_{i}$ for all $i$, and $e_{i}=n /\left(n / e_{i-1}, s_{i}\right) \quad\left(e_{0}=1\right)$,

(v) $e_{r}=e_{r-1}$ if $s_{r}=0$.

Let $f_{i}=f_{i+1} f_{i}^{\prime}$. The number of basic irreducible unitary representations $\pi$ in general position whose associated sequence is $\left(s_{1}, e_{1}, f_{1}\right), \ldots,\left(s_{r}, e_{r}, f_{r}\right)$ is

$$
\left(q^{f_{r}}-1\right) \frac{n}{f_{r}^{2}} \prod_{i=1}^{r}\left(\sum_{d \mid f_{i}^{\prime}} q^{f_{i-1} d} \mu\left(f_{i}^{\prime} / d\right)\right) q^{\alpha}, \quad \alpha=\sum_{j=1}^{r} f_{i}\left[\frac{\left(s_{i}-s_{i-1}-1\right) e_{i}}{n}\right],
$$

if $s_{r}>0$

$$
\frac{n}{f_{r}^{2}} \prod_{i=1}^{r}\left(\sum_{d \mid f_{i}^{\prime}} q^{f_{i-1} d} \mu\left(f_{i}^{\prime} / d\right)\right) q^{\alpha}, \quad \alpha \text { as above, }
$$

if $s_{r}=0 \quad\left(f_{0}=1, s_{r+1}=-1, f_{i}^{\prime}=f_{i} / f_{i-1}\right.$, and [ ] is the greatest integer function), and all these representations are of dimension

$$
f_{r} \frac{q^{n}-1}{q^{n / e_{r}}-1} q^{\gamma}, \quad \gamma=\frac{n}{2}\left[-1+\frac{f_{r}}{n_{r}}+\sum_{i=1}^{r} s_{i}\left(\frac{1}{n_{i-1}}-\frac{1}{n_{i}}\right)\right], n_{i}=e_{i} f_{i} .
$$

Proof. Begin by considering how many characters $\chi$ have $\left(s_{1}, e_{1}, f_{1}\right)$ as the first associated triple. We must have

$$
\chi\left(1+\gamma \varpi^{s_{1}}\right)=\psi \circ \operatorname{Tr}_{k_{n / k}} \alpha \gamma^{\sigma^{-s_{1}}},
$$

where $\alpha \varpi^{-s_{1}}$ generates a field of ramification index $e_{1}$ and residue class degree $f_{1}$. The ramification index is automatic, from (iv). For the residue class degree, we need to have $N_{k_{n} / k_{n / e_{1}}} \alpha$ generating $k_{f_{1}}$. If $N_{k_{n} / k_{n / e_{1}}} \alpha_{1}=N_{k_{n} / k_{n / e_{1}}} \alpha_{2}$, then $\alpha_{1} \varpi^{-s_{1}}$ and $\alpha_{2} \varpi^{-s_{1}}$ are conjugate by an element of $k_{n}$, so that we need only count the elements in $k_{n / e_{1}}$ generating $k_{f_{1}}$. There are $\sum_{d \mid f_{1}} \mu\left(f_{1} / d\right) q^{d}=Q\left(f_{1}\right)$ such $\alpha$. Each is in a conjugacy class of $f_{1}$ elements under $D^{\times} / K$.

For each $j$ with $s_{2}<j<s_{1}$ and $\left(n / e_{1}\right) \mid j$, we have a choice of $q^{f_{1}}$ ways of extending $\chi$. For the other $j$ with $s_{2}<j<s_{1}$, we have no choice in how to extend $\chi$. Thus there are $\left(1 / f_{1}\right) q^{f_{1}\left[\left(s_{1}-s_{2}-1\right) e_{1} / n\right]} Q\left(f_{1}\right)$ distinct $\chi$ with $\left(s_{1}, e_{1}, f_{1}\right)$ as the first associated triple and no further triple before $s_{2}$.

At $s_{2}$, we need to have $\alpha_{2} \eta_{1}^{-s_{2}}$ generate a field of ramification index $e_{2}$ and residue class degree $f_{2}$ over $F$. The $e_{2}$ part is automatic, from (iv). For $f_{2}$, it is better to think of $\alpha_{2} \eta_{1}^{-s_{2}}$ generating a field of residue class degree $f_{2}^{\prime}$ 
over $F\left[\eta_{1}\right]$. As before, there are $\sum_{d \mid f_{2}^{\prime}} \mu\left(f_{2} / f_{1} d\right) q^{f_{1} d}=Q\left(f_{1}, f_{2}\right)$ such $\alpha$, mod conjugacy by $k_{n / e_{1}}$. Each is in a conjugacy class of $f_{2}^{\prime}$ elements under the group of elements commuting with $\chi$.

Proceeding inductively, we get the number of characters on $K^{1}$ to be

$$
f_{r}^{-1} \prod_{i=1}^{r}\left(\sum_{d \mid f_{i}^{\prime}} q^{f_{i-1} d} \mu\left(f_{i}^{\prime} / d\right)\right) q^{\alpha}, \quad \alpha=\sum_{j=1}^{r} f_{i}\left[\left(s_{i}-s_{i-1}-1\right) e_{i} / n\right],
$$

provided that $s_{r}>0$. If $s_{r}=0$, the above formula holds with the sum and product to $r-1$ instead of $r$. For the number of characters on $K$, we need to multiply by $q^{f_{r}}-1$ if $s_{r}>0$ and by $\sum_{d \mid f_{r}^{\prime}} \mu\left(f_{r}^{\prime} / d\right) q^{f_{r-1} d}$ if $s_{r}=0$. Finally, there are $n / f_{r}$ further extensions of our character $\chi$ to the group of elements $\eta_{r}^{f_{r}}$. We thus get (a).

As for the dimension, assume first that $s_{i}$ is odd for all $i$. Then we can see by induction that $\pi$ is induced from a character $\chi$ on the group $H=$ $K^{s_{1}^{\prime}}\left(D_{1}^{\times} \cap K^{s_{2}^{\prime}}\right) \cdots\left(D_{r-1} \cap K^{s_{r}^{\prime}}\right) D_{r}^{\times}$. We compute its index in $D^{\times}$. Obviously, we can work modulo $K^{s_{1}^{\prime}}$ and modulo $\varpi^{n}$. We have

$$
\left[H \cap K^{s_{2}^{\prime}}: K^{s_{1}^{\prime}}\right]=q^{\beta_{1}}, \quad \beta_{1}=\left[\frac{s_{1}^{\prime}-s_{2}^{\prime}}{f_{1}}\right] \frac{n}{e_{1}},
$$

since $H \cap K^{s_{2}^{\prime}}$ contains elements $1+\gamma \varpi^{j}+\cdots, s_{2}^{\prime} \leq j<s_{1}^{\prime}$, only if $f \mid j$, and there are then $q^{n / e_{1}}$ choices for $\gamma$. Since $s_{1}^{\prime}$ and $s_{2}^{\prime}$ are both odd and divisible by $f_{2}$, we also have $\beta_{1}=\left[s_{1}-s_{2}\right] n / 2 n_{1}$.

Similar reasoning (note that $s_{r} \neq 0$ ) gives

$$
\left[H \cap K^{s_{i}^{\prime}}: H \cap K^{s_{i-1}^{\prime}}\right]=q^{\beta_{i}}, \quad \beta_{i}=\left[s_{i-1}-s_{i}\right] n / 2 n_{i},
$$

for $1 \leq i<r$. We also have

$$
\left[H \cap K^{1}: H \cap K^{s_{r}^{\prime}}\right]=q^{\beta_{r}}, \quad \beta_{r}=\left[\frac{\left(s_{r}^{\prime}-1\right)}{f_{r}}\right] \frac{n}{e_{r}} .
$$

But $2 s_{r}^{\prime}-1=s_{r}$ is divisible by $f_{r}$. If $2 s_{r}^{\prime}-1=c_{r} f_{r}$, then

$$
\left[\frac{2 s_{r}^{\prime}-1}{f_{r}}\right]=\left[\frac{c_{r}}{2}\right]=\frac{s_{r}-f_{r}}{2 f_{r}} .
$$

Hence $\beta_{r}=\left(s_{r}-s_{r+1}\right) n / 2 n_{r}$, where (for this part of the proof) $s_{r+1}=f_{r}$.

We also have $\left[H \cap K: H \cap K^{1}\right]=q^{n / e_{r}}-1$ and $\left[H:(H \cap K)\left\langle\varpi^{n}\right\rangle\right]=n / f_{r}$. Multiplying gives

$$
\left[H: K^{s_{1}^{\prime}}\left\langle\varpi^{n}\right\rangle\right]=\frac{n}{f_{r}} q^{\beta}\left(q^{n / e_{r}}-1\right), \quad \beta=\sum_{i=1}^{r} \beta_{i} .
$$


Hence

$$
\begin{aligned}
\operatorname{dim} \pi & =\left[D: K^{s_{1}^{\prime}}\left\langle\varpi^{n}\right\rangle\right] /\left[H: H^{s^{\prime}}\left\langle\varpi^{n}\right\rangle\right] \\
& =n\left(q^{n}-1\right) q^{\left(s_{1}^{\prime}-1\right) n} /\left[H: H^{s_{1}^{\prime}}\left\langle\varpi^{n}\right\rangle\right] \\
& =f_{r} \frac{q^{n}-1}{q^{n / e_{r}}-1} q^{n\left(s_{1}^{\prime}-1\right)-\beta} .
\end{aligned}
$$

From here, it is a question of putting $n\left(s_{1}^{\prime}-1\right)-\beta$ in some more pleasant form. Set $n_{i}=e_{i} f_{i}, n_{0}=e_{0}=f_{0}=1$. We have

$$
\begin{aligned}
n\left(s_{1}^{\prime}-1\right)-\beta & =\frac{n}{2}\left(s_{1}-1\right)-\sum_{i=1}^{r} \frac{\left(s_{i}-s_{i+1}\right) n}{2 n_{i}} \\
& =-\frac{n}{2}+\frac{n}{2} \sum_{i=1}^{r} s_{i}\left[\frac{1}{n_{i-1}}-\frac{1}{n_{i}}\right]+n f_{r} / n_{r} \\
& =\frac{n}{2}\left[-1+\frac{f_{r}}{n_{r}}+\sum_{i=1}^{r} s_{i}\left(\frac{1}{n_{i-1}}-\frac{1}{n_{i}}\right)\right] .
\end{aligned}
$$

This gives (b) when all $s_{j}$ are odd. If, say, $s_{1}$ is even, then we have

$$
\left[H \cap K^{s_{2}^{\prime}}: K^{s_{1}^{\prime \prime}}\right]=q^{\beta_{1}}, \quad \beta_{1}=\left[\frac{s_{1}^{\prime \prime}-s_{2}^{\prime}}{f_{1}}\right] \frac{n}{e_{r}} .
$$

Here, since $s_{1}^{\prime \prime}=s_{1} / 2$, we get

$$
\beta_{1}=\left(s_{1}-s_{2}-f\right) n / 2 n_{1} .
$$

But the Weil representation of $\S 4$ corresponding to $s_{1}$ has dimension $q^{n\left(1-e_{1}\right) / 2}$, while $\left[D: K^{s_{1}^{\prime \prime}}\left\langle\varpi^{n}\right\rangle\right]=n\left(q^{n}-1\right) q^{\left(s_{1}^{\prime \prime}-1\right) n}$. The effect is that (5.6) is unchanged. A similar analysis shows that the formula holds regardless of the parity of the $s_{j}$. (The analysis is carried out in more detail in $\S 3$ of [7].)

6

It may be useful to see how the construction of representations presented in this paper compares with the constructions in previous cases. When $n=p$, we necessarily have $r_{1}=1$, and the situation is relatively simple. For $n=p^{2}$ or $p n_{0}$ (with $\left.\left(p, n_{0}\right)=1\right)$, the construction presented here seems to be more straightforward than those in [4] and [6].

There remains the case $(p, n)=1$. The basic simplification is that in (3.13), for instance, we can choose $\beta$ to be in $k$, since $\operatorname{Tr}_{k_{n} / k}$ is faithful on $k$. Therefore $\alpha_{i+1}^{\prime} \eta$ commutes with $\eta$. The same statement applies to (3.15). The result is that we may always assume that $E_{(j)} \subseteq E_{(j+1)}$. (In particular, $E_{(j)}=E_{(j+1)}$ unless $j$ is one of the $s_{i}$.) Furthermore, $\chi$ is nontrivial on $E_{\left(s_{1}\right)}^{\times} \cap K^{s_{1}}$, and, similarly, $\tilde{\chi_{\left(s_{j}\right)}}$ is nontrivial on $E_{\left(s_{j}\right)}^{\times} \cap K^{s_{j}}$ (see $\S 3$ for notation). This simplifies the structure of the satisfactory extension $H$ of Proposition 4.1. 
Let $E=E_{\left(s_{r}\right)}$, where $\left(s_{1}, e_{1}, f_{1}\right), \ldots,\left(s_{r}, e_{r}, f_{r}\right)$ are the triples associated to the representation $\chi$. Then $\left.\chi\right|_{E^{\times}}$has the property that $\left.\chi\right|_{K^{s_{1}+1}}$ is trivial; $\left.\chi\right|_{K^{s_{1}}}$ and $\left.\chi\right|_{K^{s_{2}+1}}$ factor through $N_{E / E_{\left(s_{1}\right)}}$, but not through any norm map $N_{E / E^{\prime}}$ with $E_{\left(s_{1}\right)} \supsetneqq E^{\prime} ;\left.\chi\right|_{K^{s_{2}}}$ and $\left.\chi\right|_{K^{s_{3}+1}}$ factor through $N_{E / E_{\left(s_{2}\right)}}$, but not through any norm map $N_{E / E^{\prime}}$ with $E_{\left(s_{2}\right)} \supsetneqq E^{\prime}$, and so on. This leads to the Howe factorization of $\chi$ (see [12] or [17]), which gives a definition of the $s_{i}$ depending only on $\left.\chi\right|_{E^{\times}}$. In the general case, the triples $\left(s_{i}, e_{i}, f_{i}\right)$ give a weak analogue of the Howe factorization.

A formula for $\operatorname{dim} \pi$ is given as Theorem 3.25 of [7] for the case where $(n, p)=1$. We repeat it here, changing notation slightly so that it does not conflict with the notation in this paper. Let $n_{i}=e_{i} f_{i}$, and let $a=n / n_{r}$, $m_{i}=n_{r} / n_{i}$, and $j_{i}=1+s_{i} / a f_{r}$. Then

$$
\operatorname{dim} \pi=f_{r} \frac{q^{n}-1}{q^{n / e_{r}}-1} q^{\left(a f_{r} / 2\right)\left[a \alpha(\theta)+a+1-a n_{r}-e_{r}\right]},
$$

where

$$
\alpha(\theta)=\sum_{i=1}^{r} j_{i}\left(m_{i-1}-m_{i}\right), \quad m_{0}=n_{r} .
$$

The definition of $j_{i}$ has the advantage of fitting in naturally with the definition of the conductors of the terms in the Howe factorization. The formula was written to involve $a$ so that it would be easy to see the change in $\operatorname{dim} \pi$ as $n$ (the degree of the division algebra) varied, while the character $\left.\chi\right|_{E}$ remained fixed. Some algebra shows that (6.1) agrees exactly with Theorem 5.5. Thus the dimension formula of [7] has a generalization to division algebras of arbitrary degree over $F$.

In this section we give an example of the construction of supercuspidals. We let $F=\mathbf{F}_{3}[[X]]$ and $n=27$. Then $\varpi^{27}=X$ and $k=\mathbf{F}_{3}$. Let $\sigma$ be the Frobenius, $\gamma \mapsto \gamma^{3}$. We examine the representations associated with the sequence of triples

$$
(45,3,1), \quad(33,9,1), \quad(19,27,1) .
$$

Since the $s_{i}$ (namely 45, 33, and 19) are all odd, we avoid the Heisenberg construction.

We use the notation of [6] to describe $k_{27}=\mathbf{F}_{327}$. A basis for $k_{27} / k$ is constructed as follows: define $\alpha_{1}, \alpha_{2}, \alpha_{3}$ by

$$
\alpha_{1}^{3}=\alpha_{1}+1, \quad \alpha_{2}^{3}=\alpha_{2}+\alpha_{1}^{2}, \quad \alpha_{3}^{3}=\alpha_{3}+\alpha_{2}^{2} .
$$

Next define $\beta_{j}(0 \leq j \leq 26)$ as follows: if $j=9 a_{3}+3 a_{2}+a_{1} \quad\left(0 \leq a_{i} \leq 2\right)$, then $\beta_{j}=\alpha_{3}^{a_{3}} \alpha_{2}^{a_{2}} \alpha_{1}^{a_{1}}$. The $\beta_{j}$ form the desired basis.

We define $\psi$ on $F$ by

$$
\psi\left(\Sigma \gamma_{j} X^{j}\right)=\psi\left(\gamma_{0}\right)=e^{2 \pi i \gamma_{0} / 3} \quad\left(\gamma_{0} \in \mathbf{Z} / 3 \mathbf{Z}\right) .
$$


Now we begin to construct $\chi$. On $K^{45}$ we must have

$$
\chi\left(1+\gamma \varpi^{45}\right)=\psi \circ \operatorname{Tr}_{D / K}\left(\delta_{-45} \varpi^{-45} \cdot \gamma \varpi^{45}\right),
$$

where $F\left[\delta_{-45} \varpi^{-45}\right]$ is a totally ramified extension of degree 3 . Then $\left(\delta_{-45} \varpi^{-45}\right)^{3}$ $=\gamma_{-45} \varpi^{-135}=\gamma_{-45} X^{-5}$, where $\gamma_{-45} \in k^{\times}$, and we may assume after conjugation that $\delta_{-45}= \pm 1 \in k$. For definiteness, take $\delta_{-45}=1$, so that $\chi\left(1+\gamma \varpi^{45}\right)=\psi \circ \operatorname{Tr}_{k_{n} / k} \gamma^{\sigma^{-45}}=\psi \circ \operatorname{Tr}_{k_{n} / k} \gamma$. Then we may set $\eta_{(45)}=\varpi$. Notice that $E_{(45)}=F\left[\varpi^{9}\right]$.

We next extend $\chi$ to $K^{44}, K^{43}, \ldots$ For $K^{44}$, we know that the extension $\chi_{0}$ of Lemma 3.8 is unique, and it is then clear that

$$
\chi_{0}(1+x)=\psi \circ \operatorname{Tr}_{D / K}\left(\varpi^{-45} x\right) .
$$

It is also not hard to check that if $\chi$ is not conjugate to $\chi_{0}$, so that

$$
\chi \cdot \chi_{0}^{-1}(1+x)=\psi \circ \operatorname{Tr}_{D / K}\left(\delta_{-44}^{\prime} \varpi^{-44} x\right), \quad \operatorname{Tr}_{k_{27} / k_{3}}\left(\delta_{-44}^{\prime}\right)=\delta_{-44} \neq 0,
$$

then $s_{2}=44$. Therefore $\chi=\chi_{0}$ on $K^{44}$. A similar argument shows that $\chi=\chi_{0}$ on $K^{37}$, where $\chi_{0}$ is still given by (7.1). We also have $\eta_{(44)}=\cdots=$ $\eta_{(37)}=\varpi$ and $E_{(37)}=\cdots=E_{(45)}$.

On $K^{36}$, however, we have more leeway. We need

$$
\chi \cdot \chi_{0}^{-1}(1+x)=\psi \circ \operatorname{Tr}_{D / K}\left(\delta_{-36}^{\prime} \eta_{(37)}^{(-36)} x\right), \quad \operatorname{Tr}_{k_{27} / k_{9}}\left(\delta_{-36}^{\prime}\right)=\delta_{-36},
$$

where $\delta_{-36} \eta_{(37)}^{-36} \in E_{(37)}$. This is true if $\delta_{-36} \in k$. From Corollary 2 of Proposition 2.1 in [6], we may assume that $\delta_{-36}^{\prime}$ is a multiple of $\beta_{18}$. If, for example, $\delta_{-36}^{\prime}=\beta_{18}$, then

$$
\eta_{(36)}=\eta_{(37)}\left(1+\delta \varpi^{9}\right)
$$

where $\delta=\beta_{26}+\cdots$ can be calculated as in (3.10). We shall instead take the easy way out and assume that $\delta_{-36}=0$. Then we may also take $\delta_{-36}^{\prime}=0$, and $\eta_{(36)}=\eta_{(37)}=\varpi, E_{(36)}=E_{(37)}$.

On $K^{35}$ and $K^{34}$, we also assume that $\chi=\chi_{0}$, where $\chi_{0}$ is given by (7.1); $\eta_{(36)}=\eta_{(34)}$ and $E_{(36)}=E_{(34)}$. At $K^{33}$, however, we have a jump index. We must have

$$
\chi \cdot \chi_{0}^{-1}(1+x)=\psi \circ \operatorname{Tr}_{D / K}\left(\delta_{-33}^{\prime} \eta_{(34)}^{-33} x\right), \quad \operatorname{Tr}_{k_{27} / k_{9}}\left(\delta_{-33}^{\prime}\right)=\delta_{-33},
$$

where $\delta_{-33} \eta_{(34)}^{-33}=\delta_{-33} \varpi^{-33}$ generates a totally ramified field of degree 3 over $E_{1}=E_{(34)}$. Since $\left(\delta_{-33} \varpi^{-33}\right)^{3}=\gamma_{-33} \varpi^{-99}$ and $\varpi^{-99} \in E_{1}$, we must have $\gamma_{-33} \in k^{\times}$. By conjugation, we may then assume that $\delta_{-33} \in k^{\times}$. We take $\delta_{-33}=1$. Then $\delta_{-33}^{\prime}=\beta_{18}+$ any linear combination of $\beta_{0}, \ldots, \beta_{17}$, as a computation (using results from $\S 2$ of [6]) shows. 
We now need $\eta_{(33)}=\eta_{1}\left(1+\delta \varpi^{12}\right)$, where $\eta_{1}=\eta_{(34)}$. Given a choice of $\delta_{-33}^{\prime}$, we could compute $\delta$ and $\eta_{(33)}$ as in (3.10). We use another device here. We have

$$
\chi(1+x)=\psi \circ \operatorname{Tr}_{D / K}\left[\left(\varpi^{-45}+\delta_{-33}^{\prime} \varpi^{-33}\right) x\right], \quad x \in P^{33} .
$$

So $\eta_{(33)}$ must commute with $\varpi^{-45}+\delta_{-33}^{\prime} \varpi^{-33} \bmod P^{-32}$, or (equivalently) with $X^{2}\left(\varpi^{-45}+\delta_{-33}^{\prime} \varpi^{-33}\right)=\varpi^{9}+\delta_{-33}^{\prime} \varpi^{21} \bmod P^{22}$. So we choose $\delta_{-33}^{\prime}$ to make $\eta_{(33)}^{9} \equiv \varpi^{9}+\delta_{-33}^{\prime} \varpi^{21} \bmod P^{22}$. With $\delta=\beta_{26}, \eta_{(33)}^{9}$ is of the desired form (though computing $\delta_{-33}^{\prime}$ is an unpleasant task). Then $\eta_{(33)}=\varpi+\beta_{26}^{3} \varpi^{13}$, and $E_{(33)}=F\left[\eta_{(33)}^{3}\right]$.

As before, we must have $\chi=\chi_{0}$ on $K^{32}$ and $K^{31}$ (at least after conjugating), so that $\chi$ is given on $K^{31}$ by (7.2). On $K^{30}$,

$$
\chi \cdot \chi_{0}^{-1}(1+x)=\psi \circ \operatorname{Tr}_{D / K}\left(\delta_{-30}^{\prime} \eta_{(31)}^{-30} x\right), \quad \eta_{(31)}=\eta_{(33)},
$$

where $\delta_{-30}=\operatorname{Tr}_{k_{27} / k_{3}} \delta_{-30}^{\prime}$ is such that $\delta_{-30} \eta_{(31)}^{30} \in E_{(31)}$. Therefore $\delta_{-30} \in k$, and we may take $\delta_{-30}^{\prime}$ to be any linear combination of $\beta_{0}, \ldots, \beta_{24}$. We shall take $\delta_{-30}^{\prime}=0$. We have similar choices at $K^{27}$ and $K^{24}$, and we will assume that $\delta_{27}=0$ and $\delta_{24}=1$. Then $\eta_{(25)}=\cdots=\eta_{(33)}$ and

$$
\begin{gathered}
\chi(1+x)=\psi \circ \operatorname{Tr}_{D / K}\left[\left(\varpi^{-45}+\delta_{-33}^{\prime} \eta_{(34)}^{-33}+\delta_{-24}^{\prime} \eta_{(25)}^{-24}\right) x\right], \quad x \in P^{24} ; \\
\eta_{(24)}=\eta_{(25)}\left(1+\delta_{1} \eta_{1}^{9}\right)\left(1+\delta_{0} \varpi^{21}\right), \quad \delta_{1} \in k_{9}, \delta_{0} \in k_{27} .
\end{gathered}
$$

As in the analysis for $K^{33}$, we can finesse the computations by requiring that $\eta_{(24)}^{3} X^{-1} \equiv \varpi^{-45}+\delta_{-33}^{\prime} \eta_{(34)}^{-33}+\delta_{-24}^{\prime} \eta_{(25)}^{-24} \bmod P^{-23}$, where $\operatorname{Tr}_{k_{27} / k_{3}} \delta_{-24}^{\prime}=1$. Setting $\delta_{1}=\beta_{8}$ and $\delta_{0}=-\beta_{26}$ fixes an appropriate $\delta_{24}^{\prime}$. Then $E_{(24)}=F\left[\eta_{(24)}^{3}\right]$.

For $K^{23}$, we again have $\chi=\chi_{0}$ and $\eta_{(23)}=\eta_{(24)}$, while $\chi$ is given by (7.3). We now go on to $H_{22}=K^{23}\left(D_{1}^{\times} \cap K^{22}\right)$, where $D_{1}=\left\{\Sigma \gamma_{j} \varpi^{j}: \gamma_{j} \in k_{9}\right\}$. We can no longer describe $\chi$ as before, since $H_{22}$ is not obviously isomorphic to an additive subgroup of $D$. We could compute a central character $\chi^{\sim}$ of $D_{1}^{\times}$ agreeing with $\chi$ on $D_{1}^{\times} \cap K^{34}$ and then consider $\chi\left(\chi^{\sim}\right)^{-1}$ on $D_{1}^{\times}$, but that seems to be fairly complicated. We instead consider the extension $\chi_{0}$ to $H_{22}$. (One can verify in other ways that $\chi_{0}\left(1+\gamma \varpi^{22}\right)=1$ for all $\gamma \in k_{9}$.) Since $s_{3} \neq 22, \chi=\chi_{0}$ on $H_{22}$ (at least after conjugacy), so that $\eta_{(22)}=\eta_{(23)}$ and $E_{(22)}=E_{(23)}$.

On $H_{21}=K^{23}\left(D_{1}^{\times} \cap K^{21}\right)$, we have a choice of extensions of $\chi$. We need

$$
\chi \cdot \chi_{0}^{-1}\left(1+\gamma \eta_{(22)}^{-21}\right)=\psi \circ \operatorname{Tr}_{k_{9} / k}\left(\delta_{21}^{\prime} \gamma^{3}\right),
$$


where $\delta_{-21}=\operatorname{Tr}_{k_{9} / k_{3}} \delta_{-21}^{\prime}$ is such that $\delta_{-21} \eta_{(22)}^{-21} \in E_{(22)}$. Therefore we need $\delta_{-21} \in k$, and $\delta_{-21}^{\prime}$ must be a linear combination of $\beta_{0}, \ldots, \beta_{6}$. We will take $\delta_{-21}^{\prime}=0$, so that $\eta_{(21)}=\eta_{(22)}$ and $E_{(21)}=E_{(22)}$.

On $H_{20}=K^{23}\left(D_{1}^{\times} \cap K^{20}\right), \chi=\chi_{0}$ (after conjugating) and $\eta_{(20)}=\eta_{(21)}$, $E_{(20)}=E_{(21)}$. On $H_{19}=K^{23}\left(D_{1}^{\times} \cap K^{19}\right), \chi \neq \chi_{0}$ because $s_{3}=19$. We must have

$$
\chi \cdot \chi_{0}^{-1}\left(1+\gamma \eta_{(20)}^{19}\right)=\psi \circ \operatorname{Tr}_{k_{9} / k}\left(\delta_{-19}^{\prime} \gamma^{\sigma^{-19}}\right),
$$

where $\delta_{-19}=\operatorname{Tr}_{k_{9} / k_{3}} \delta_{-19}^{\prime}$ is such that $E_{(19)}\left[\delta_{-19} \eta_{(20)}^{-19}\right]$ is totally ramified of degree 27 over $F$. This is in fact true for any nonzero $\delta_{-19}$, but there is no loss in assuming that $\delta_{-19}= \pm 1$, since the other choices are conjugate to these. We will choose $\delta_{-19}=-1$. Some calculation shows that

$$
\eta_{(19)}=\eta_{(20)}\left(1-\alpha_{6} \eta_{(33)}^{14}\right)
$$

commutes with $\chi$ for an appropriate $\delta_{-19}^{\prime}$. We have $E_{(19)}=F\left[\eta_{(19)}\right]$. Let $\eta_{(20)}=\eta_{2}, E_{(20)}=E_{2}$, etc.

For $H_{18}=K^{23}\left(D_{1}^{\times} \cap K^{18}\right)$, we have $\chi \cdot \chi_{0}^{-1}\left(1+\gamma \eta_{(19)}^{18}\right)=\psi \circ \operatorname{Tr}_{k_{9} / k}\left(\delta_{-18}^{\prime} \gamma^{\sigma^{-18}}\right)$, where $\delta_{-18}=\operatorname{Tr}_{k_{9} / k} \delta_{-18}^{\prime}$ is such that $\delta_{-18} \eta_{(19)}^{-18} \in E_{(19)}$. This holds for all $\delta_{-18}^{\prime}$, but there is no loss of generality in assuming that $\delta_{-18}^{\prime}$ is a multiple of $\beta_{8}$. We will assume that $\delta_{-18}^{\prime}=0$, so that $\chi=\chi_{0}$ and $\eta_{(18)}=\eta_{(19)}$. We will assume similarly that $\eta_{(17)}=\eta_{(19)}$.

We now go to $H_{16}=K^{23}\left(K^{17} \cap D_{1}^{\times}\right)\left(K^{16} \cap D_{2}^{\times}\right)$and compare $\chi$ with $\chi_{0}$ there:

$$
\chi \cdot \chi_{0}^{-1}\left(1+\gamma \eta_{(17)}^{16}\right)=\psi \circ \operatorname{Tr}_{k_{3} / k}\left(\delta_{-16}^{\prime} \gamma^{\sigma^{-16}}\right),
$$

where $\delta_{-16}=\operatorname{Tr}_{k_{3} / k} \delta_{-16}^{\prime}$ is such that $\delta_{-16} \eta_{(17)}^{-16} \in E_{(17)}$. This is automatic, but we may take $\delta_{-16}^{\prime}$ to be a multiple of $\beta_{2}$. We will assume that $\delta_{-16}^{\prime}=\beta_{2}$. Then

$$
\eta_{(16)}=\eta_{(17)}\left(1+\beta_{2} \eta_{2}\right)
$$

as a calculation shows. We also have $E_{(16)}=F\left[\eta_{(16)}\right]$. We have similar choices for $H_{15}, \ldots, H_{10}=K^{23}\left(K^{17} \cap D_{1}^{\times}\right)\left(K^{10} \cap D_{2}^{\times}\right)$; we will assume that at each step $\chi=\chi_{0}$. Then $\eta_{(16)}=\cdots=\eta_{(10)}$ and $E_{(16)}=\cdots=E_{(10)}$.

We now have a satisfactory extension. So $\eta_{(10)}=\eta_{3}$ and $E_{(10)}=E_{3}$. In our case, $E_{3}=D_{3}$. We extend $\chi$ to a character of $H=H_{10} E_{3}^{\times}$, which we also call $\chi$, and induce to get $\pi$.

\section{REFERENCES}

1. C. Bushnell and A. Fröhlich, Gauss sums and p-adic division algebras, Lecture Notes in Math., no. 987, Springer, Berlin and New York, 1983.

2. H. Carayol, Représentations cuspidales du groupe linéaire, Ann. Sci. École Norm. Sup. (4) 17 (1984), 191-226. 
3. L. Corwin, Representations of division algebras over local fields, Adv. in Math. 13 (1974), 259-267.

4. _ Representations of division algebras over local fields. II, Pacific J. Math. 101 (1982), 49-70.

5. __ Approximation of prime elements in division algebras over local fields and unitary representations of the multiplicative group, Pacific J. Math. 130 (1987), 223-251.

6. __ Supercuspidal representations of $\mathrm{GL}_{p p^{\prime}}(F), F$ p-adic, preprint.

7. L. Corwin, A. Moy, and P. J. Sally, Jr., Degrees and formal degrees for division algebras and $\mathrm{GL}_{n}$ over a p-adic field, preprint.

8. P. Deligne, D. Kazhdan, and M. F. Vigneras, Représentations des algébras centrales simples p-adiques, Représentations des Groupes Réductif sur un Corps Local, Hermann, Paris, 1984, pp. 33-117.

9. P. Gerardin, Construction de series discrétes p-adiques, Lecture Notes in Math., no. 462, Springer, Berlin and New York, 1972.

10. R. Howe, Representation theory for division algebras over local fields (tamely ramified case), Bull. Amer. Math. Soc. 77 (1971), 1063-1066.

11. __ Kirillov theory for compact p-adic groups, Pacific J. Math. 73 (1977), 365-381.

12. __ Tamely ramified supercuspidal representations of $\mathrm{GL}_{n}$, Pacific J. Math. 73 (1977), 437459.

13. H. Koch, Eisenteinsche Polynomfolgen and Arithmetik in Divisionsalgebren über lokalen Körpern, Math. Nachr. 104 (1981), 229-251.

14. __, Zur Arithmetik in Divisionsalgebren über lokalen Körpern, Math. Nachr. 100 (1981), 9-19.

15. P. Kutzko and D. Manderscheid, On the supercuspidal representations of $\mathrm{GL}_{N}, N$ the product of two primes, 1988, preprint.

16. G. W. Mackey, Induced representations of groups, Amer. J. Math. 73 (1951), 576-592.

17. A. Moy, Local constants and the tame Langlands correspondence, Amer. J. Math. 108 (1986), 863-930.

18. A. Weil, Basic number theory, Springer, Berlin and New York, 1967.

Department of Mathematics, Rutgers University, New Brunswick, NJ 08903 